\title{
A moist aquaplanet variant of the Held-Suarez test for atmospheric model dynamical cores
}

\author{
Diana R. Thatcher and Christiane Jablonowski \\ Department of Climate and Space Sciences and Engineering, University of Michigan, 2455 Hayward, \\ Ann Arbor, MI 48109, USA \\ Correspondence to: Diana R. Thatcher(dtatch@umich.edu) \\ Received: 16 July 2015 - Published in Geosci. Model Dev. Discuss.: 29 September 2015 \\ Revised: 28 January 2016 - Accepted: 28 February 2016 - Published: 4 April 2016
}

\begin{abstract}
A moist idealized test case (MITC) for atmospheric model dynamical cores is presented. The MITC is based on the Held-Suarez (HS) test that was developed for dry simulations on "a flat Earth" and replaces the full physical parameterization package with a Newtonian temperature relaxation and Rayleigh damping of the low-level winds. This new variant of the HS test includes moisture and thereby sheds light on the nonlinear dynamics-physics moisture feedbacks without the complexity of full-physics parameterization packages. In particular, it adds simplified moist processes to the HS forcing to model large-scale condensation, boundary-layer mixing, and the exchange of latent and sensible heat between the atmospheric surface and an ocean-covered planet. Using a variety of dynamical cores of the National Center for Atmospheric Research (NCAR)'s Community Atmosphere Model (CAM), this paper demonstrates that the inclusion of the moist idealized physics package leads to climatic states that closely resemble aquaplanet simulations with complex physical parameterizations. This establishes that the MITC approach generates reasonable atmospheric circulations and can be used for a broad range of scientific investigations. This paper provides examples of two application areas. First, the test case reveals the characteristics of the physics-dynamics coupling technique and reproduces coupling issues seen in full-physics simulations. In particular, it is shown that sudden adjustments of the prognostic fields due to moist physics tendencies can trigger undesirable large-scale gravity waves, which can be remedied by a more gradual application of the physical forcing. Second, the moist idealized test case can be used to intercompare dynamical cores. These examples demonstrate the versatility of the MITC approach and suggestions are made for further application areas. The new moist variant of the HS test can be considered a test case of intermediate complexity.
\end{abstract}

\section{Introduction}

Atmospheric general circulation models (GCMs) are important tools for understanding the climate system. However, as climate simulations advance with the use of ever more complex models there is a profound need to understand the physical characteristics of GCMs in idealized setups (Held, 2005). This evaluation hierarchy should include 2-D shallowwater models, dry 3-D dynamical cores, moist idealized 3D dynamical cores, full-physics aquaplanet configurations (Neale and Hoskins, 2000), GCMs with complex physical parameterizations and prescribed surface conditions (Gates et al., 1999), Earth system models of intermediate complexity (EMICS) (Claussen et al., 2002; Weber, 2010) and fully coupled atmosphere-ocean-ice-land Earth system models (ESMs). This quest for a model hierarchy reveals that no standard simplified moist evaluation technique has been established yet. Only recently, Frierson et al. (2006), Frierson (2007b), O'Gorman and Schneider (2008), and Reed and Jablonowski (2012) introduced GCM configurations with highly simplified, moist physical parameterizations. The paper falls into this category and describes a moist dynamical core test of intermediate complexity for climate-like studies. Throughout this paper the term "dynamical" core refers to the resolved fluid flow on the computational grid. In contrast, the physical parameterizations (or "physics") represent all subgrid-scale processes that cannot be resolved explicitly, such as precipitation and radiation.

Differences in GCMs are apparent when comparing models with different parameterizations, computational grids, and numerical methods (e.g., Lauritzen et al., 2010; Blackburn et al., 2013). However, the interactions and feedbacks between the dynamical core and physical parameterizations make it difficult to diagnose sources of error or clearly distin- 
guish between causes and effects. The large uncertainties associated with the physical parameterizations may contribute to, or even hide, biases originating within the dynamical core. Systematic methods for evaluating and comparing GCMs are therefore paramount for model development. In an ideal situation, dynamical core tests should evaluate the fluid flow by directly comparing the model results to a known analytic solution. However, analytical solutions are not available for complex simulations and can only be used to evaluate very idealized flow conditions, such as steady states (Jablonowski and Williamson, 2006), linear flow regimes (Baldauf et al., 2014), or the advection of passive tracers with prescribed wind fields (Kent et al., 2014). Dynamical core tests for more complex, nonlinear flow scenarios without a known solution rely on the premises that models tend to converge toward a high-resolution reference solution and the results of multiple dynamical cores closely resemble each other within some uncertainty limit (Jablonowski and Williamson, 2006).

A well-established climate-focused evaluation method for dry 3-D dynamical cores is the Held-Suarez (HS) test with idealized physical parameterizations, namely, a Newtonian temperature relaxation and Rayleigh damping of low-level winds (Held and Suarez, 1994). This test neither contains moisture nor a seasonal or diurnal cycle, and the surface geopotential is flat. Nevertheless, HS-driven simulations resemble the general circulation of the atmosphere. There is no analytic solution to the HS test. Therefore, model intercomparisons are generally used to check if the HS results are reasonable compared to other GCMs. The HS test has been shown to be sensitive to spatial resolution (Jablonowski, 1998; Wan et al., 2008) and has been useful for explaining differences in climate models without the need for complex physical parameterizations or surface boundary conditions (Chen et al., 1997; Zhang et al., 2013).

A variety of studies have utilized the HS test and variations thereof. The HS test is often used to validate the statistical behavior of new dynamical cores (Smolarkiewicz et al., 2001; Fournier et al., 2004; Tomita and Satoh, 2004; Richardson et al., 2007) and for dynamical core intercomparisons (Jablonowski, 1998). In addition, Wedi and Smolarkiewicz (2009) applied amplified HS forcings within a small-planet testing framework for intercomparisons of nonhydrostatic dynamical cores. Polvani and Kushner (2002) used the HS forcing with a slightly modified equilibrium temperature profile and a high model top to explore the extratropical tropospheric response to imposed stratospheric temperature perturbations. The HS test was also used by Yao and Jablonowski $(2013,2015)$ to analyze a quasi-biennial oscillation (QBO)-like circulation in the tropical stratosphere. Furthermore, Mitchell et al. (2002) applied HS forcings with topography to understand the minimum ensemble size and error growth of a data assimilation algorithm. Galewsky et al. (2005) paired the HS test with passive moisture tracers, though the moist tracers did not release latent heat. Two moist extensions of the HS test were described by Grabowski and Smolarkiewicz (2002) and Kurowski et al. (2015), who relaxed the water vapor mixing ratio towards specified relative humidity values. However, their method was not focused on mimicking the Earth's atmospheric flow conditions, as we do in this paper, but instead only demonstrated the use of an idealized test in understanding the characteristics of new numerical methods with moisture feedbacks. Note that the latter two publications do not describe the moist HS extensions in detail so their experimental setup cannot be easily replicated.

All HS application examples described above demonstrate the usefulness of idealized GCM assessments and a simpleto-use definition of the test case. We highlight that these HS examples go well beyond the initial intent of the HS publication, which solely focused on a proposal for dynamical core intercomparisons. In this paper, we propose a slightly modified variant of the HS forcing and include moisture processes via very few simplified physical parameterizations on a water-covered planet similar to Reed and Jablonowski (2012) (herein referred to as RJ12), such as some bulk aerodynamic latent and sensible heat fluxes at the surface, a simple boundary-layer mixing of temperature and moisture, and large-scale precipitation.

Our test is more complex than the moist HS variants in Grabowski and Smolarkiewicz (2002) and Kurowski et al. (2015), yet less complex than the simplified physics package in Frierson et al. (2006) or O'Gorman and Schneider (2008). The latter two suggested the use of radiative fluxes instead of the thermal HS relaxation, a slab ocean with constant depth instead of a prescribed sea surface temperature (SST), and a more complicated Monin-Obukhov-type boundarylayer parameterization. However, we show that our proposed moist variant of the HS test is capable of simulating a quasirealistic climate and closely mimics the characteristics of full-physics aquaplanet "CONTROL" simulations as defined in Neale and Hoskins (2000). Such traditional aquaplanet setups utilize a complex-physics package on an ocean-covered Earth with analytically prescribed SSTs and equinoctial radiation. Since land-atmosphere interactions and mountain effects are removed, it makes it easier to discern causes and effects in idealized process studies. As a consequence, aquaplanet simulations have become a valuable tool for evaluating and comparing different combinations of dynamical cores, model designs, parameter settings, and physical parameterizations (e.g., Williamson and Olson, 2003; Medeiros et al., 2008; Williamson, 2008b; Mishra et al., 2011b; Rauscher et al., 2013). We envision similar broad application areas for our suggested moist variant of the HS test, which is accompanied by the Fortran source code (see the Supplement). This guarantees the ease-of-use and makes the experimental setup and results reproducible.

This paper has three goals. First, we explain the design of the moist idealized test case (MITC), which is easy to use and implement. Second, we provide MITC example results that were generated with the spectral element (SE) dynamical core (Taylor and Fournier, 2010; Dennis et al., 2012) 
of the Community Atmosphere Model (CAM5) version 5.3 (Neale et al., 2010). This model is under development at the National Center for Atmospheric Research (NCAR) and various Department of Energy (DoE) laboratories. The climate patterns of the moist idealized circulation are discussed and directly compared to their CAM5-SE full-physics aquaplanet counterparts. This demonstrates that the general circulations in both approaches are comparable and that the test case leads to reasonable climatic conditions. Third, we present two example application areas for the moist idealized test case to shed light on its versatility. They include selected snapshots of a dynamical core intercomparison that involves the four CAM5 dynamical cores: SE, finite volume (FV), Eulerian (EUL) spectral transform, and semi-Lagrangian (SLD) spectral transform (Neale et al., 2010). Furthermore, we demonstrate that the MITC approach exposes intricacies of the physics-dynamics coupling strategy that cannot be revealed in dry HS experiments. The MITC is computationally efficient and easily ported to different dynamical cores, allowing other modeling groups to assess their own GCMs and test the characteristics of new dynamical cores. Thus, the MITC can serve as a valuable tool for understanding and improving dynamical cores and their physics-dynamics interplay. Furthermore, the MITC can be employed for idealized climate studies, as mentioned above, that improve our theoretical understanding of the general circulation.

This paper is structured as follows. Section 2 contains the description of the moist idealized physics processes. The four CAM5 dynamical cores are briefly described in Sect. 3. Section 4 discusses the general circulation of the MITC CAM5SE simulations compared to their aquaplanet counterparts. Section 5 provides insight into two example applications. In particular, the physics-dynamics coupling strategy in the SE dynamical core is analyzed, and snapshots of a MITC dynamical core intercomparison are presented. Section 6 makes further suggestions for other application areas and possible extensions of the MITC approach. Furthermore, this section calls for community participation to foster the moist dynamical core research. Section 7 summarizes all findings.

\section{Description of the physical parameterizations of the moist idealized test case}

The proposed MITC approach utilizes simplified moist physics parameterizations paired with slightly modified forcings from the HS test (Held and Suarez, 1994). The simplified moist physics parameterizations follow those from the short-term tropical cyclone test case by RJ12 with several modifications. In brief, the physical forcings incorporate surface fluxes of latent and sensible heat as well as momentum, boundary-layer mixing, large-scale precipitation, and radiation. Here, we briefly describe the key equations and processes, and point to RJ12 and Held and Suarez (1994) for some of the details of the implementation. In particular, im- plicit time-stepping approaches are used to enhance the numerical stability of the surface flux and boundary-layer calculations. These details are also shown in the supplementary Fortran routine, which allows for a rapid inclusion of the MITC in other dynamical cores.

The MITC modifications of RJ12 and HS, which are detailed below, make the simplified moist physics package appropriate for long-term climate studies. Neither seasonal cycles nor topography are included; therefore, the climate statistics should be identical in both hemispheres. Any hemispheric differences in the model results are due to sample size rather than dynamical processes. We recommend spinning the model up from an idealized moist initial state (see Appendix A) for 6 months and analyzing the following 30 months ( $\sim 2.5$ years) of data. In our simulations, 1 month always contains 30 days and is independent of the actual calendar months.

\subsection{Large-scale precipitation}

Moisture is removed from the atmosphere using the largescale condensation scheme described in RJ12; see Eqs. (1) through (14) in that publication. Condensation occurs when the grid cell reaches saturation according to the ClausiusClapeyron equation. The saturation-specific humidity, $q_{\mathrm{sat}}$, is

$q_{\mathrm{sat}}(T)=\frac{\varepsilon}{p} e_{0}^{*} \exp \left[-\frac{L}{R_{\mathrm{v}}}\left(\frac{1}{T}-\frac{1}{T_{0}}\right)\right]$,

where $p$ is the pressure of the moist air with units $\mathrm{Pa}$ and $T$ is the temperature with units Kelvin. The constants are defined as $\varepsilon=0.622, T_{0}=273.16 \mathrm{~K}$ is the triple point of water, $e_{0}^{*}=610.78 \mathrm{~Pa}$ is the saturation vapor pressure at $T_{0}$, $L=2.5 \times 10^{6} \mathrm{~J} \mathrm{~kg}^{-1}$ is the latent heat of vaporization at $T_{0}$, and $R_{\mathrm{V}}=461.5 \mathrm{~J} \mathrm{~kg}^{-1} \mathrm{~K}^{-1}$ is the gas constant for water vapor. The condensation rate, $C$, is given by

$C=\frac{\mathrm{d} q_{\mathrm{sat}}}{\mathrm{d} t}=\frac{1}{\Delta t}\left(\frac{q-q_{\mathrm{sat}}(T)}{1+\frac{L}{c_{p}} \frac{L q_{\mathrm{sat}}}{R_{\mathrm{v}} T^{2}}}\right)$,

where $\Delta t$ denotes the physics time step, $q$ is the specific humidity, and $c_{p}=1004.6 \mathrm{~J} \mathrm{~kg}^{-1} \mathrm{~K}^{-1}$ is the specific heat at constant pressure. If a dynamical core uses a leapfrog timestepping scheme $\Delta t$ needs to be replaced with $2 \Delta t$ as pointed out in RJ12. Note that Eq. (2) is only strictly valid under the assumption that there is no change in the pressure of the moist air, which is typical for physical parameterizations in hydrostatic models. However, Eq. (2) in combination with the isobaric temperature adjustment shown in Eq. (3) can also be used as an anelastic approximation in nonhydrostatic models (Thurre and Laprise, 1996; Malardel, 2011). This is further detailed in Sect. 2.6.

If $q>q_{\mathrm{sat}}$ (over $100 \%$ relative humidity), then latent heat is released and the condensate is immediately recorded as precipitation and removed from the system; thus, reevaporation does not occur and there are no clouds. The 
resulting temperature and specific humidity changes due to condensation at constant pressure are

$$
\begin{aligned}
& \frac{\partial T}{\partial t}=\frac{L}{c_{p}} C, \\
& \frac{\partial q}{\partial t}=-C .
\end{aligned}
$$

The condensation leads to the large-scale precipitation rate $P_{\mathrm{ls}}$ with units in meters (of water column) per second

$$
P_{\mathrm{ls}}=\frac{1}{\rho_{\text {water }} g} \int_{0}^{p_{\mathrm{s}}} C \mathrm{~d} p \approx \frac{1}{\rho_{\text {water }} g} \sum_{k=1}^{\text {nlev }} C_{k}\left(p_{k+1 / 2}-p_{k-1 / 2}\right),
$$

where $\rho_{\text {water }}=1000 \mathrm{~kg} \mathrm{~m}^{-3}$ is the density of water, $g=$ $9.80616 \mathrm{~m} \mathrm{~s}^{-2}$ is gravity, and $p_{\mathrm{s}}$ denotes the moist surface pressure in $\mathrm{Pa}$. The precipitation is summed over all nlev vertical model levels where $C_{k}$ is the condensation rate at model level $k$, and $p_{k \pm 1 / 2}$ is the pressure of the moist air in $\mathrm{Pa}$ at the interface between two full model levels. In Eq. (5) it is assumed that the level index $k$ increases downward (i.e., $k=1$ is the top model level) such that the difference between the two interface levels, or pressure level thickness, is always positive.

\subsection{Prescribed boundary conditions}

The MITC approach is designed for a water-covered Earth without topography. Therefore, the surface geopotential, $\Phi_{\mathrm{s}}$, needs to be set to zero. The constant SST from RJ12 is replaced with a prescribed SST profile dependent on latitude. The SST profile, $T_{\mathrm{s}}$, is defined by

$T_{\mathrm{s}}=\Delta T \exp \left(-\frac{\phi^{2}}{2(\Delta \phi)^{2}}\right)+T_{\min }$,

where $\Delta T=29 \mathrm{~K}$ is the SST difference between the Equator and poles, $T_{\min }=271 \mathrm{~K}$ is the SST at the poles, $\phi$ is the latitude in radians, and $\Delta \phi=26 \pi / 180$ controls the latitudinal width of the Gaussian function. This SST profile was motivated by lowest-level temperature profiles from a dry HS experiment. It also resembles the prescribed SSTs from the APE CONTROL experiment (Neale and Hoskins, 2000), particularly in the tropics and midlatitudes. The SST profile includes temperatures slightly below freezing polewards of $60^{\circ} \mathrm{N} / \mathrm{S}$. However, these temperatures do not drop below $271 \mathrm{~K}$, the approximate freezing point of sea water. Thus, the prescribed SST acts as a lower-boundary forcing on the atmosphere to facilitate reasonable latent and sensible heat fluxes.

\subsection{Surface fluxes}

The original HS Rayleigh friction of the zonal and meridional winds at the lowest model level, shown later in Eq. (14), acts as the surface momentum flux. Therefore, the RJ12 formulation for the zonal and meridional surface momentum forcings (Eqs. 33 and 34 in RJ12) is not used to avoid double counting the surface friction. However, surface fluxes of sensible and latent heat are still needed. These temperature and moisture surface fluxes come from the RJ12 specification (see their Eqs. 22 and 23 in kinematic units with the time-implicit update Eqs. C3 and C6). They lead to the surface forcings,

$$
\begin{aligned}
\frac{\partial T_{\mathrm{a}}}{\partial t} & =\frac{C_{H}\left|\boldsymbol{v}_{\mathrm{a}}\right|\left(T_{\mathrm{s}}-T_{\mathrm{a}}\right)}{z_{\mathrm{a}}} \\
\frac{\partial q_{\mathrm{a}}}{\partial t} & =\frac{C_{E}\left|\boldsymbol{v}_{\mathrm{a}}\right|\left(q_{\mathrm{sat}, \mathrm{s}}-q_{\mathrm{a}}\right)}{z_{\mathrm{a}}},
\end{aligned}
$$

at the lowest model level. If a model needs surface fluxes in energy units $\left(\mathrm{W} \mathrm{m}^{-2}\right)$, the corresponding sensible $(H)$ and latent $(E)$ heat formulations at the surface are

$$
\begin{aligned}
& H=\rho_{\mathrm{a}} c_{p} C_{H}\left|\boldsymbol{v}_{\mathrm{a}}\right|\left(T_{\mathrm{s}}-T_{\mathrm{a}}\right) \\
& E=\rho_{\mathrm{a}} L C_{E}\left|\boldsymbol{v}_{\mathrm{a}}\right|\left(q_{\mathrm{sat}, \mathrm{s}}-q_{\mathrm{a}}\right) .
\end{aligned}
$$

In these equations $T_{\mathrm{a}}, q_{\mathrm{a}}$, and $\rho_{\mathrm{a}}$ are the temperature, specific humidity, and density of the moist air at the lowest model level, respectively, and $q_{\mathrm{sat}, \mathrm{s}}$ is the saturation-specific humidity at the surface with temperature $T_{\mathrm{s}}$. The unitless bulk transfer coefficients for sensible heat, $C_{H}=0.0044$, and water vapor, $C_{E}=0.0044$, are set to the same value. These values are 4 times higher than the values used for the tropical cyclone studies in RJ12 to enhance the planetary boundary-layer mixing and surface fluxes. This is motivated by the fact that the typical HS lowest-level wind speed $\left|\boldsymbol{v}_{\mathrm{a}}\right|$ is weak in comparison to the tropical cyclone wind speeds in RJ12. Note though that these $C_{H}$ and $C_{E}$ settings are bigger than theoretical values derived from observations. These typically range between 0.001 and 0.0025 (e.g., Pond et al., 1974; Smedman et al., 2007) depending on the environmental conditions. Our enhanced values can therefore be viewed as a compensation mechanism for the missing complexity of the physical mixing and surface-exchange processes.

The lowest model level wind speed, $\left|\boldsymbol{v}_{\mathrm{a}}\right|$, and height position, $z_{\mathrm{a}}$, are

$\left|\boldsymbol{v}_{\mathrm{a}}\right|=\sqrt{u_{\mathrm{a}}^{2}+v_{\mathrm{a}}^{2}}$
$z_{\mathrm{a}}=\frac{R_{\mathrm{d}} T_{\mathrm{v}, \mathrm{a}}}{g} \frac{\left(\ln p_{\mathrm{s}}-\ln p_{-}\right)}{2}$,

where $R_{\mathrm{d}}=287.04 \mathrm{~J} \mathrm{~kg}^{-1} \mathrm{~K}^{-1}$ is the dry-air gas constant, $T_{\mathrm{v}, \mathrm{a}}=T_{\mathrm{a}}\left(1+0.608 q_{\mathrm{a}}\right)$ is the virtual temperature at the lowest model level, and $p_{-}$is the pressure of the moist air in $\mathrm{Pa}$ at the edge (interface level) between the lowest and second lowest full model levels. The definition of $z_{\mathrm{a}}$ in Eq. (12) corrects a sign error in Eq. (28) of RJ12, where $p_{\mathrm{S}}$ and $p_{-}$were accidentally reversed. In our vertical grid configuration detailed later, the position of the lowest model level, $z_{\mathrm{a}}$, is located at a height of approximately $60-65 \mathrm{~m}$. 
The MITC temperature forcing can also be modified for different prognostic variables. For example, if a model solves the thermodynamic equation in terms of potential temperature then the sensible heat forcing at the surface (Eq. 7) can be reformulated to

$$
\frac{\partial \Theta_{\mathrm{a}}}{\partial t}=\frac{C_{H}\left|\boldsymbol{v}_{\mathrm{a}}\right|\left(T_{\mathrm{s}}-T_{\mathrm{a}}\right)}{z_{\mathrm{a}}}\left(\frac{p_{00}}{p_{\mathrm{a}}}\right)^{\frac{R_{\mathrm{d}}}{c_{p}}},
$$

where $\Theta_{\mathrm{a}}$ and $p_{\mathrm{a}}$ denote the potential temperature and pressure of the moist air at the lowest model level, respectively, and $p_{00}=10^{5} \mathrm{~Pa}$ is a reference pressure. The derivation of this equation implicitly assumes that the pressure of the moist air stays constant in time in the physical parameterization package. As mentioned in Sect. 2.1, this is a typical assumption in hydrostatic GCMs and is further discussed for hydrostatic and nonhydrostatic models in Sect. 2.6.

\subsection{Boundary-layer mixing}

The original HS Rayleigh damping of low-level winds acts as the boundary-layer mixing scheme for the horizontal velocity fields. The horizontal velocity vector, $v=(u, v)^{T}$, with the zonal and meridional wind components $u$ and $v$, respectively, is damped by the HS formulation

$$
\frac{\partial \boldsymbol{v}}{\partial t}=-k_{f} \max \left(0, \frac{\sigma-\sigma_{b}}{1-\sigma_{b}}\right) \boldsymbol{v},
$$

where $k_{f}=1 \mathrm{day}^{-1}, \sigma_{b}=0.7$, and $\sigma=p / p_{\mathrm{s}}$ is the vertical sigma coordinate where the pressures $p$ and $p_{\mathrm{s}}$ have the same units. This Rayleigh damping affects the boundary layer below approximately $700 \mathrm{hPa}$. The velocity damping is strong at the lowest model level where it acts as surface friction as mentioned in Sect. 2.3. The HS Rayleigh friction is used instead of the boundary-layer mixing of momentum described in RJ12 (their Eqs. 15, 16, 40, 41, and 46).

In practice, all of our example CAM5 dynamical cores utilize hybrid pressure-based vertical coordinates with $p\left(\eta, p_{\mathrm{s}}\right)=a(\eta) p_{00}+b(\eta) p_{\mathrm{s}}$, where $\eta$ represents each vertical level (Simmons and Burridge, 1981). $\eta$ is approximately equivalent to $\sigma$ in the lower troposphere because the $a(\eta)$ coefficients are typically zero or small below $700 \mathrm{hPa}$ (e.g., see Table B1 in RJ12 for the values of the CAM5 $a$ and $b$ hybrid coefficients at level interfaces). Therefore, we use $\eta$ instead of $\sigma$ in our implementation of Eq. (14). The findings in our paper do not depend on this choice because the corresponding climate statistics are indistinguishable.

The HS boundary-layer momentum forcing, Eq. (14), provides sufficient damping for the $u$ and $v$ fields, but does not affect the temperature and specific humidity. RJ12 suggest a simple planetary boundary-layer (PBL) turbulence parameterization in which the vertical turbulent flux of potential temperature, $\overline{w^{\prime} \Theta^{\prime}}$, and vertical turbulent flux of specific humidity, $\overline{w^{\prime} q^{\prime}}$, are

$\overline{w^{\prime} \Theta^{\prime}}=-K_{E} \frac{\partial \Theta}{\partial z}$, $\overline{w^{\prime} q^{\prime}}=-K_{E} \frac{\partial q}{\partial z}$,

where $\Theta$ is the potential temperature, $w$ is the vertical velocity, and $z$ is the height. The overbar indicates a time average and the prime denotes the deviation from the time average.

The eddy diffusivity coefficient, $K_{E}$, used in Eqs. (15) and (16) is set to

$K_{E}= \begin{cases}C_{E}\left|\boldsymbol{v}_{\mathrm{a}}\right| z_{\mathrm{a}} & \text { for } p>p_{\mathrm{pbl}}, \\ C_{E}\left|\boldsymbol{v}_{\mathrm{a}}\right| z_{\mathrm{a}} \exp \left(-\left[\frac{p_{\mathrm{pbl}}-p}{p_{\text {strato }}}\right]^{2}\right) & \text { for } p \leq p_{\mathrm{pbl}},\end{cases}$

where $p_{\mathrm{pbl}}=850 \mathrm{hPa}$ is the top of the boundary layer and $p_{\text {strato }}=100 \mathrm{hPa}$ impacts the rate of decrease of the boundary-layer mixing with height. RJ12 explains in detail how the PBL physics tendencies for temperature and specific humidity are applied (see their Eqs. 18, 38, 39, and their Appendix D, especially the semi-implicit update Eqs. D28, D29, and D31). These details are also included in the supplementary Fortran routine.

\subsection{Radiation}

Idealized radiation is based on the HS Newtonian temperature relaxation to a prescribed radiative equilibrium temperature, which is a function of latitude and pressure. The prescribed equilibrium temperature profile has been slightly modified from the original HS profile in order to facilitate a model solution that is similar to the zonally and temporally averaged climatologies of aquaplanet simulations. The original HS equilibrium temperature leads to a climatology that is too warm and energetic in comparison to aquaplanet simulations and observations. The modified profile for the equilibrium temperature, $T_{\mathrm{eq}}$, is

$$
\begin{gathered}
T_{\mathrm{eq}}(\phi, p)=\max \left\{200 \mathrm{~K},\left[T_{\mathrm{Equator}}-(\Delta T)_{y} \sin ^{2} \phi-\right.\right. \\
\left.\left.(\Delta \theta)_{z} \log \left(\frac{p}{p_{0}}\right) \cos ^{2} \phi\right]\left(\frac{p}{p_{0}}\right)^{\kappa}\right\},
\end{gathered}
$$

where $(\Delta T)_{y}=65 \mathrm{~K},(\Delta \theta)_{z}=10 \mathrm{~K}, p_{0}=1000 \mathrm{hPa}$ is a reference pressure, $p$ is the pressure of the moist air in $\mathrm{hPa}$, and $\kappa=R_{\mathrm{d}} / c_{p}$. The two differences in comparison to the original HS $T_{\text {eq }}$ profile are the use of $T_{\text {Equator }}=294 \mathrm{~K}$ in Eq. (18) instead of the original HS equatorial maximum temperature $315 \mathrm{~K}$, and the redefinition of the meridional Equator-pole temperature difference $(\Delta T)_{y}=65 \mathrm{~K}$ instead of the original HS value of $60 \mathrm{~K}$.

The model temperature, $T$, is relaxed toward the equilibrium temperature profile by

$\frac{\partial T}{\partial t}=-k_{T}(\phi, \sigma)\left[T-T_{\mathrm{eq}}(\phi, \sigma)\right]$

at each grid point and physics time step. The temperature relaxation coefficient, $k_{T}$, is defined as

$k_{T}(\phi, \sigma)=k_{\mathrm{a}}+\left(k_{\mathrm{s}}-k_{\mathrm{a}}\right) \max \left(0, \frac{\sigma-\sigma_{b}}{1-\sigma_{b}}\right) \cos ^{4} \phi$, 
where $k_{\mathrm{a}}=1 / 40 \mathrm{day}^{-1}$ and $k_{\mathrm{s}}=1 / 4 \mathrm{day}^{-1}$, as in the original HS test. As before, we use $\eta$ instead of $\sigma$ in our implementation of Eq. (20).

\subsection{Physics-dynamics coupling}

The MITC approach allows for the analysis of dynamical cores, often with vastly different numerical techniques and computational grids, in the presence of moisture. In our implementation the large-scale condensation and precipitation are computed first, followed by the forcing from the surface fluxes of temperature and moisture. Boundary-layer mixing of temperature and moisture is applied next, followed by the Rayleigh friction mechanism of the original HS forcing. Simplified radiation, represented by the modified HS temperature relaxation (Sect. 2.5), is computed last. The surface fluxes and boundary-layer mixing of temperature and moisture are implemented using a partially implicit time-stepping scheme (see Eqs. C3, C6, and D23-D31 in RJ12). The Rayleigh friction is applied with a forward-Euler time-stepping approach, although a fully implicit time-stepping scheme is also possible if desired for enhanced numerical stability. The results do not depend on this choice. These implementation details and the corresponding Fortran routine are provided to enable other modelers to replicate this setup. All physical forcings are implemented in a sequential (a.k.a. time-split) way. This means that each process uses updated state variables before the next physical forcing is computed. Therefore, the order of the physics processes matters and should not be modified.

The actual coupling between the dynamical core and the physics package is model dependent (Williamson, 2002) and defaults should be used. For example, the dynamical cores SE and FV are designed with the time-split physicsdynamics coupling strategy, which means that the dynamical core has already updated the prognostic variables before the physical package is entered. In contrast, EUL and SLD employ a parallel (a.k.a. process-split) coupling strategy. These two dynamical cores use the same state variables for both the computation of the dynamical and physical forcing tendencies, and apply these tendencies together at the end of the time step to update the prognostic variables.

The simplified physics package presented here assumes that the sequence of physical processes does not change the mass of the moist air; e.g., the pressure of the moist air is unchanged while going through the physics sequence. This is also assumed in the complex CAM5 physics package, and is a standard in hydrostatic GCMs. However, once moisture is added or removed via the physical parameterizations the pressure of the moist air or the moist air density needs to change in models that use these in their dynamical core formulations. It is paramount to implement this pressure or density adjustment while conserving the dry air mass at the very end of each physics time step. An example implementation for CAM5-FV is shown in Neale et al. (2010) (their Sect. 3.1.8). However, the implementation algorithm is model dependent and might also be represented by a global "mass-fixing" algorithm in some models, such as EUL and SLD. In addition, the geopotential needs to be recomputed in hydrostatic models with pressure-based vertical coordinates after the temperature and pressure adjustments from the physical parameterizations. This is typically done within the dynamical core via the integration of the hydrostatic equation and should be checked.

We note that, strictly speaking, nonhydrostatic models with height-based vertical coordinates need to utilize a constant density (a.k.a constant volume) assumption within the physical parameterization package. However, as explained by Thurre and Laprise (1996), Thurre (1998), and Malardel (2011) the assumption of a constant pressure of the moist air in the physics combined with an isobaric update of the temperature (Eq. 3) corresponds to an anelastic filtering of the diabatic forcing in nonhydrostatic models. In particular, Malardel (2011) showed, with a nonhydrostatic and hydrostatic version of the same GCM, that this anelastic physicsdynamics coupling in nonhydrostatic simulations leads to almost identical results in comparison to hydrostatic simulations at hydrostatic scales. This ensures that the isobaric physics processes described in this paper are also applicable (as an approximation) to nonhydrostatic model formulations with grid spacings wider than about $10 \mathrm{~km}$. If unapproximated physics-dynamics coupling strategies are desired in nonhydrostatic GCMs, careful attention needs to be paid to the model design. As explained by Petrik et al. (2011) (their Eq. 23), models with a prognostic thermodynamic equation in the isochoric $c_{\mathrm{V}} T$ form and a prognostic equation for the pressure of the moist air need physics adjustments due to latent heat and mass redistributions of water species in both prognostic equations. On the other hand, nonhydrostatic models that use the thermodynamic equation in potential temperature form and a continuity equation for the dry air density can be coupled in an identical way for both isobaric and isochoric assumptions (William Skamarock, NCAR, personal communication, 2015). However, an isochoric requirement invalidates the saturation adjustment shown in Eq. (2), which will need to be replaced with an iterative procedure. Such changes to the MITC test case are allowed for nonhydrostatic models but will need to be documented. However, as argued above the current physics-dynamics coupling is applicable as an anelastic approximation in nonhydrostatic models and such isochoric changes should only marginally affect the simulation results at hydrostatic scales.

In the four CAM5 example dynamical cores discussed below, the physical tendencies and the pressure corrections of the moist air are applied as a sudden adjustment after each physics time step. This is an obvious choice for models with identical dynamics and physics time steps such as the CAM5 dynamical cores EUL and SLD. It is also the default setting for CAM5-FV, which sub-cycles the dynamical core multiple times before the physical forcings abruptly adjust the prognostic variables. However, the sub-cycled CAM5-SE dy- 
namical core provides two coupling options that are both explored in this paper. Besides the sudden adjustment of the state variables after each long physics time step (denoted as se_ftype $=1$ later), the alternative option adds a fraction of the physics forcing at each sub-cycle, and thereby short, dynamics time step (denoted as se_ftype $=0$ in Sect. 5.1). The latter leads to a gentler adjustment of the prognostic variables.

\section{Brief description of the four CAM5 dynamical cores}

We illustrate the characteristics of the moist idealized test case via four example dynamical cores. As mentioned before, these are the SE, FV, EUL spectral transform, and SLD spectral transform dynamical cores of CAM5, which is the atmosphere component of the Community Earth System Model (CESM). All dynamical cores use 30 pressure-based vertical levels with the model top at about $2 \mathrm{hPa}$ (see Appendix B in RJ12 for the exact level placement). In addition, all CAM5 dynamical cores are built upon the hydrostatic "primitive equation" set. Detailed descriptions of all four CAM5 dynamical cores can be found in Neale et al. (2010). Here we only present a brief description of them.

The SE dynamical core (Dennis et al., 2012) is the most recent dynamical core available in CAM and is considered the new default in future versions of CAM. SE is based upon on a cubed-sphere grid with a co-located Arakawa Agrid staggering of all prognostic variables. The cubed-sphere grid eliminates the "pole problem" caused by the converging meridians in latitude-longitude grids and increases scalability on massively parallel computer systems. The horizontal discretization uses a continuous Galerkin spectral finite element method, or spectral element method, and is fourthorder accurate in the horizontal direction (Taylor et al., 1997; Taylor and Fournier, 2010). The vertical discretization employs a floating Langrangian coordinate in which the prognostic variables are periodically remapped to a vertical reference grid. Tracers are transported via the same spectralelement scheme. The time discretization uses an explicit Runge-Kutta time-stepping method. The dynamical core is sub-cycled multiple times before the physical parameterizations are invoked. In our SE version (summer of 2013), the default physics-dynamics coupling was set to se_ftype $=1$, which uses a sudden adjustment of the state variables after each long physics time step.

The FV dynamical core is the default dynamical core for CAM versions 4 and 5. FV is built on a regular latitudelongitude grid with Arakawa D-grid staggering. The horizontal discretization is based on a mass-conserving finitevolume transport scheme with semi-Lagrangian provisions for long time steps (Lin and Rood, 1996, 1997; Lin, 2004). As in SE, the vertical discretization is built upon a floating Lagrangian coordinate with periodic vertical remapping to a reference grid. The finite-volume tracer transport in FV is inherently conservative and less diffusive than transport in EUL and SLD (Rasch et al., 2006). FV employs limiters that introduce implicit numerical diffusion. In addition, a polar Fourier filter is applied in the zonal direction poleward of about $40^{\circ} \mathrm{N} / \mathrm{S}$. FV's time-stepping method is fully explicit, and the dynamics are sub-cycled within each 2-D Lagrangian layer to guarantee the stability of the fastest waves.

The EUL spectral transform dynamical core was the default dynamical core in earlier versions of CAM and is currently available as an option within CAM5. EUL is formulated in vorticity-divergence form on an Arakawa A-grid (Neale et al., 2010). It uses a three-time-level spectral transform method on a quadratic Gaussian grid with a semiimplicit, leapfrog time integration scheme. The leapfrog scheme is stabilized via the Robert-Asselin filter with filter coefficient $\alpha=0.06$ (e.g., Jablonowski and Williamson, 2011). The vertical discretization utilizes a finite-difference method. EUL's tracer advection algorithm is built upon a semi-Lagrangian scheme.

The SLD dynamical core is an optional dynamical core in CAM5 and uses a two-time-level, semi-implicit, semiLagrangian spectral transform method as described by Neale et al. (2010). SLD utilizes a quadratic Gaussian transform grid with Arakawa A-grid staggering and a semi-Lagrangian advection algorithm for momentum, mass, and tracers with cubic interpolations. These interpolations act as numerical dissipation. In addition, SLD employs a decentering technique with the default coefficient $\epsilon=0.2$ that damps the flow field and suppresses orographic resonance in real-world applications (e.g., see Jablonowski and Williamson, 2011).

Resolutions for all four dynamical cores are approximately the same, although slight differences exist due to the underlying grids. SE uses a cubed-sphere grid with resolution ne30np4 $\left(\sim 1^{\circ}\right)$, denoting $30 \times 30$ elements across each cubed-sphere face where each element has third degree polynomials ( $\mathrm{np}=4$ collocation points) for fourth-order accuracy. FV uses a fixed latitude-longitude grid with a resolution of $1^{\circ} \times 1^{\circ}$. SE and FV have an approximate $110 \mathrm{~km}$ grid spacing at the Equator. The spectral transform models EUL and SLD apply a T85 triangular truncation and utilize a $256 \times 128$ quadratic Gaussian grid $\left(\sim 1.5^{\circ}\right)$. This corresponds to a grid spacing of about $156 \mathrm{~km}$ at the Equator. Williamson (2008b) demonstrated that these resolutions for FV and EUL are equivalent in the context of an aquaplanet simulation despite the slightly wider grid spacings in the spectral transform models. Table 1 lists the resolutions as well as the default physics and dynamics time steps for all four dynamical cores.

The CAM5 dynamical cores are paired with their default diffusion mechanisms and respective coefficients, which are also listed in Table $1^{1}$. SE utilizes an explicitly added

\footnotetext{
${ }^{1}$ For reproducibility at the $\approx 1^{\circ}$ resolutions, the CAM5-SE time step settings in the input Fortran namelist were tstep_type $=1$ (five-stage Runge-Kutta time-stepping variant), se_nsplit $=2$, and
} 
Table 1. Horizontal grid resolutions with approximate grid spacings $\Delta x$ near the Equator, physics and dynamics time steps $\Delta t$, and explicitly added diffusion mechanisms for all four dynamical cores.

\begin{tabular}{llrrrl}
\hline $\begin{array}{l}\text { Dynamical } \\
\text { Core }\end{array}$ & Resolution & $\begin{array}{r}\Delta x \\
(\mathrm{~km})\end{array}$ & $\begin{array}{r}\text { Physics } \\
\Delta t(\mathrm{~s})\end{array}$ & $\begin{array}{r}\text { Dynamics } \\
\Delta t(\mathrm{~s})\end{array}$ & $\begin{array}{l}\text { Explicitly added } \\
\text { diffusion }\end{array}$ \\
\hline SE & ne30np4 & 110 & 1800 & 300 & $\nabla^{4}$ hyper-diffusion \\
FV & $1^{\circ} \times 1^{\circ}$ & 110 & 1800 & 180 & $\nabla^{4}$ divergence damping \\
EUL & T85 & 156 & 600 & 600 & $\nabla^{4}$ hyper-diffusion \\
SLD & T85 & 156 & 1800 & 1800 & - \\
\hline
\end{tabular}

fourth-order horizontal hyper-diffusion similar to EUL, as described by Dennis et al. (2012), and the diffusion coefficients are $v=1.0 \times 10^{15} \mathrm{~m}^{4} \mathrm{~s}^{-1}$ for the rotational component and $v_{\text {div }}=(2.5)^{2} \times 10^{15} \mathrm{~m}^{4} \mathrm{~s}^{-1}$ for the divergent component. FV uses fourth-order horizontal divergence damping as further described by Whitehead et al. (2011). EUL uses an explicitly added fourth-order horizontal $\nabla^{4}$ hyperdiffusion with the coefficient $v=1.0 \times 10^{15} \mathrm{~m}^{4} \mathrm{~s}^{-1}$, as suggested by Williamson (2008a). SLD does not apply any explicitly added diffusion in the dynamical core because there is sufficient implicit dissipation due to the cubic interpolations in the semi-Lagrangian approach. This implicit numerical diffusion mimics fourth-order hyper-diffusion as assessed by McCalpin (1988).

CAM5's complex physical parameterization package is detailed by Neale et al. (2010) (see also the references therein) and is used for the aquaplanet comparisons in Sect. 4. In brief, the complex CAM5 physics package contains deep and shallow convective parameterizations, as well as a moist boundary-layer turbulence scheme based on the turbulent kinetic energy. In addition, CAM5 includes parameterizations for cloud microphysics, cloud macrophysics, surface exchanges, orographic gravity wave drag, and the radiative effects of aerosols as well as a scheme for short- and longwave radiation. Our aquaplanet simulations utilize the bulk aerosol model (BAM) with prescribed aerosols that are zonally and hemispherically symmetric.

\section{Comparison of the MITC and aquaplanet general circulations in CAM5-SE}

The simplified physics parameterizations have a different form than their complex-physics CAM5 equivalents. However, this section demonstrates that the resulting general circulation in the MITC and full-physics aquaplanet simulations is quite comparable. For brevity, we only choose CAM5-SE to characterize the general circulation and moisture charac-

rsplit $=3$, leading to a $15 \mathrm{~min}$ remapping time interval and $5 \mathrm{~min}$ dynamics and tracer time step. The Fortran namelist input setting for FV's dynamics sub-cycling method was nsplit $=10$, which sets the dynamics time step to the physics time step divided by nsplit ( $\Delta t_{\text {dyn }}=3 \mathrm{~min}$ here). The FV vertical remapping time step is equal to the physics time step. teristics in the two model configurations. The same conclusions can also be drawn when using the other CAM5 dynamical cores (not shown). Two variants of the CAM5-SE aquaplanet simulation with the "CONTROL" SST setting (Neale and Hoskins, 2000) are used for the comparison. In one simulation, the complex CAM5 physical parameterization package is used to generate a standard aquaplanet simulation. In another simulation, CAM5's Zhang-McFarlane deep convection parameterization (Zhang and McFarlane, 1995) has been turned off, leaving only large-scale precipitation as implemented in CAM5's microphysics scheme and the precipitation from CAM5's shallow convection parameterization (Park and Bretherton, 2009). The latter setup provides a more direct comparison to the MITC approach, which only includes resolved-scale (large-scale) precipitation. Throughout this paper the term aquaplanet simulation (APS) will refer to the standard aquaplanet simulation with deep convection and the aquaplanet simulation with no deep convection (APS (NDC)) will be denoted as such.

The comparison presented here demonstrates that the moist idealized physics package can create a reasonable general circulation of the moist atmosphere without the complexity of the CAM5 physics suite. Aquaplanet simulations are an attractive alternative comparison tool to observations because the new test case does not have topography or seasons, two features that greatly affect observational data. All SE simulations have the same resolution, physics and dynamics time steps, and diffusion coefficients as listed in Table 1. Unless noted otherwise, all analyses represent 2.5 year time means. However, slight latitudinal asymmetries in the means are still possible with this sample size.

\subsection{Dynamical fields and eddy components}

Figure 1a-c highlight the close resemblance between the time-mean zonal-mean temperature profiles in both the MITC and aquaplanet simulations. This is also exemplified by the similar positions of the tropopause levels (red lines), as calculated via the World Meteorological Organization (WMO) static stability criterion (WMO, 1992). The temperature cross sections are somewhat different in the lower stratosphere above $100 \mathrm{hPa}$, where the aquaplanet temperatures increase more with height. This is expected because the two aquaplanet configurations capture the radiative heating 

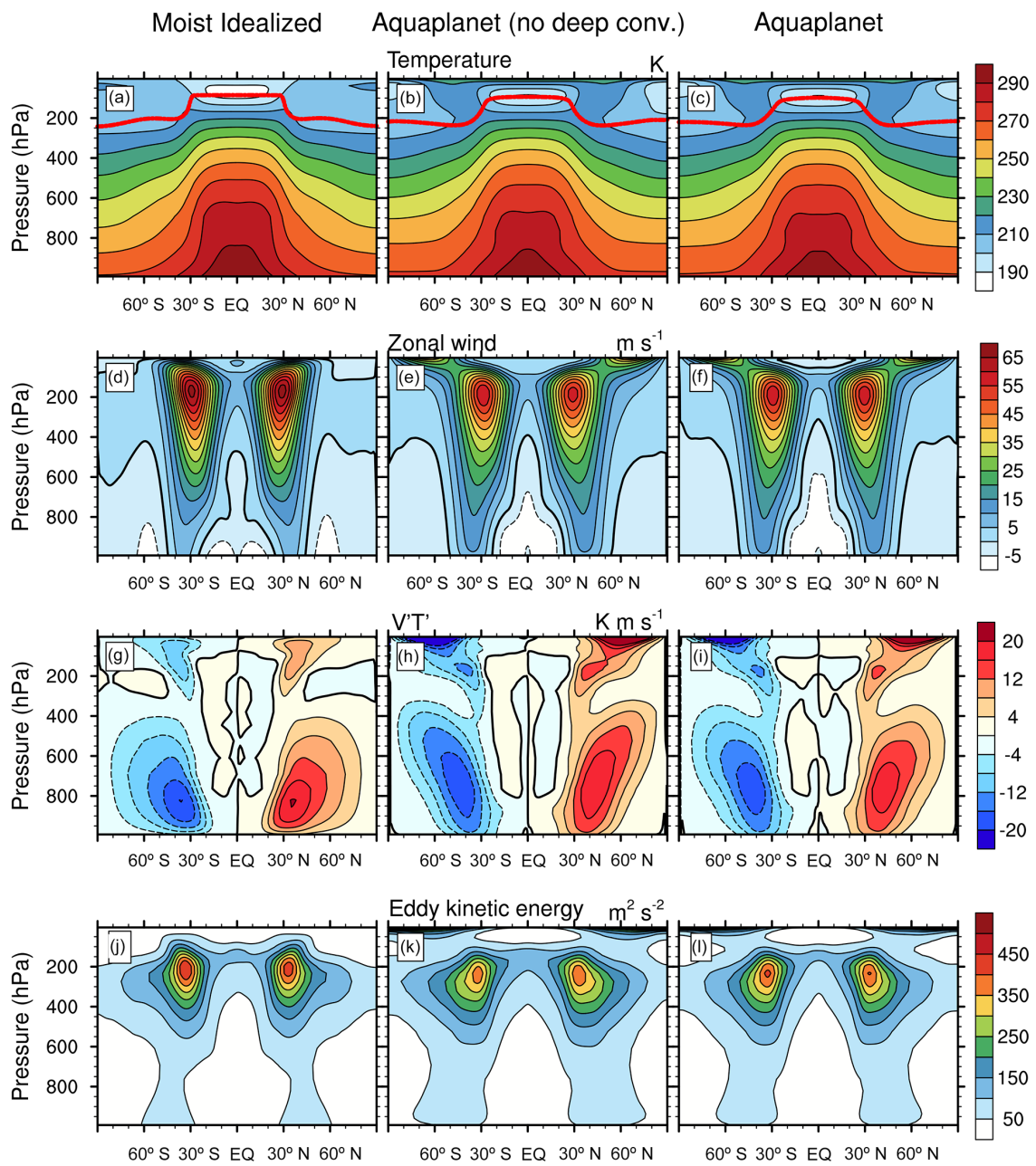

Figure 1. Comparison of CAM5-SE latitude-pressure cross sections in MITC (left column), APS (NDC) (middle column), and APS (right column): Time-mean zonal-mean $(\mathbf{a}, \mathbf{b}, \mathbf{c})$ temperature, $(\mathbf{d}, \mathbf{e}, \mathbf{f})$ zonal wind, $(\mathbf{g}, \mathbf{h}, \mathbf{i})$ meridional eddy heat flux $v^{\prime} T^{\prime}$, and $(\mathbf{j}, \mathbf{k}, \mathbf{l})$ eddy kinetic energy. The red line in $(\mathbf{a}, \mathbf{b}, \mathbf{c})$ indicates the position of the tropopause.

in the lower stratosphere. The MITC temperature profile, on the other hand, is governed by the relaxation toward the equilibrium temperature profile, which is isothermal at $200 \mathrm{~K}$ in the upper troposphere and lower stratosphere. In the following discussions we do not focus on these systematic stratospheric differences, which also appear in other diagnostics but are unimportant for the analysis here.

An interesting temperature difference between the MITC and aquaplanet simulations is the vertical extent of the warm tropical temperature dome (e.g., see the $280 \mathrm{~K}$ contour) in the lower atmosphere, which extends further up in the MITC. This is caused by different condensational heating characteristics. More specifically, the condensational heating in the MITC due to large-scale precipitation maximizes around $800 \mathrm{hPa}$ near the Equator, which is also displayed later in Sect. 4.2 (Fig. 3a). In contrast, the convection parameterizations in the complex-physics aquaplanet simulations shift the equatorial condensation peaks upward to a position near
$525 \mathrm{hPa}$ in APS (Fig. 3b). This leads to the slightly warmer temperatures in the lower-to-mid-tropical MITC atmosphere. However, the time-mean global-mean temperatures are very comparable. They are $246.34 \mathrm{~K}$ in the SE MITC simulation and $246.93 \mathrm{~K}$ in SE APS. These global-mean temperatures also closely resemble the other three CAM5 dynamical cores that all lie in the range from 246.3 to $246.6 \mathrm{~K}$ for MITC and 246.6 to $247.2 \mathrm{~K}$ for APS.

The latitude-pressure cross sections of the time-mean zonal-mean zonal wind are shown in Fig. 1d-f. The zonalwind patterns are quite similar and feature westerly jets centered around $30^{\circ} \mathrm{N} / \mathrm{S}$ with maximum wind speeds around $55-60 \mathrm{~m} \mathrm{~s}^{-1}$ at $\sim 200 \mathrm{hPa}$ in the aquaplanet setups and around $65 \mathrm{~ms}^{-1}$ at $\sim 150 \mathrm{hPa}$ in MITC. The slightly higher location and higher jet speed in MITC is closely related to the temperature differences in the upper troposphere and lower stratosphere that are linked to the zonal wind via the thermal wind relationship. All model configurations develop easterly 


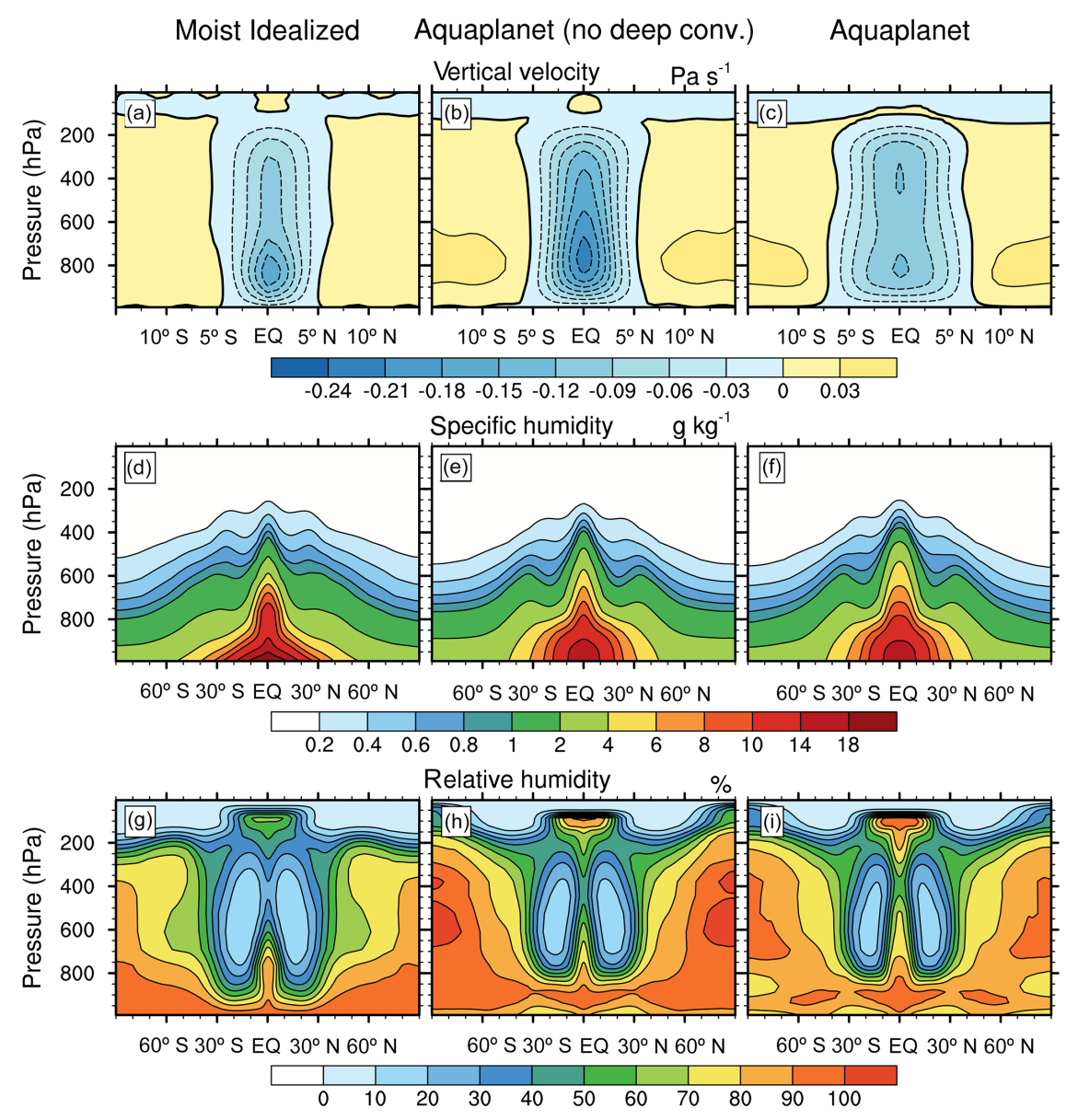

Figure 2. Comparison of CAM5-SE latitude-pressure cross sections in MITC (left column), APS (NDC) (middle column), and APS (right column): time-mean zonal-mean $(\mathbf{a}, \mathbf{b}, \mathbf{c})$ vertical pressure velocity $\omega,(\mathbf{d}, \mathbf{e}, \mathbf{f})$ specific humidity, and $(\mathbf{g}, \mathbf{h}, \mathbf{i})$ relative humidity. Note that the latitude range for $\omega$ is $15^{\circ} \mathrm{S}$ to $15^{\circ} \mathrm{N}$ and the color scale for specific humidity is nonlinear.

flows in the tropics and at high latitudes in the lower atmosphere. In addition, there is an indication that MITC and APS (NDC) (Fig. 1d and e) compare more favorably as hypothesized earlier. This is at least true in the tropics where the absence of the deep convection parameterization in APS (NDC) impacts the zonal-wind distribution most significantly.

Figure 1g-i shows the latitude-pressure cross sections of the time-mean zonal-mean meridional eddy heat flux $\overline{\left[v^{\prime} T^{\prime}\right]}$, where the primes indicate the variations from the timemeans, and the bracket and overbar symbolize the zonal and time averages, respectively. The magnitudes and the overall shapes of all three profiles compare very well. There are large areas of poleward eddy heat transport in the midlatitudes below $400 \mathrm{hPa}$, and additional midlatitudinal poleward heat transport cells above $250 \mathrm{hPa}$. All cross sections show that the poleward heat transport maximizes around $40^{\circ} \mathrm{N} / \mathrm{S}$. The main difference between the MITC and the aquaplanet simulations is the vertical extent of the eddy heat transport. The MITC eddy heat transport is strongest near $850 \mathrm{hPa}$, whereas the aquaplanet peaks are located higher up near $700 \mathrm{hPa}$. This causes the aquaplanet heat transports to be more prevalent in the region between 700 and $400 \mathrm{hPa}$ where the MITC heat transport diminishes more strongly in the upward direction.

Finally, the eddy kinetic energy profiles $\overline{\left[0.5\left(u^{\prime} u^{\prime}+v^{\prime} v^{\prime}\right)\right]}$ are shown in Fig. 1j-1. As before, the shapes and strengths of the eddy kinetic energy patterns are very similar. The MITC simulation shows the upper-tropospheric midlatitudinal peaks at a slightly higher location, which is connected to the upward shifted zonal jet maxima seen in Fig. 1d.

As a side note, the MITC and aquaplanet eddy heat fluxes and kinetic energy values match the typical values from dry HS experiments quite well with extrema around $\pm 20 \mathrm{~K} \mathrm{~m} \mathrm{~s}^{-1}$ and $400 \mathrm{~m}^{2} \mathrm{~s}^{-2}$, respectively, as shown in Wan et al. (2008). However, the presence of moisture has a profound impact on the location of the eddy kinetic energy peaks, which are shifted equatorwards by about $10^{\circ}$ in the moist simulations. Related differences are also apparent in the temperature and zonal-wind distributions. The centers of the midlatitudinal zonal jets near the tropopause shift equatorward from about 45 to $30^{\circ} \mathrm{N} / \mathrm{S}$ and the magnitudes of the zonal jets double 
from around $30 \mathrm{~m} \mathrm{~s}^{-1}$ to about $60 \mathrm{~m} \mathrm{~s}^{-1}$ in the moist simulations. These higher jet speeds are a result of the increased meridional temperature gradients throughout the troposphere (above the boundary layer) in the moist simulations. These enhanced meridional gradients are caused by tropical heating and mid-to-high-latitude cooling tendencies from the physical parameterizations, which are displayed in Fig. 3a and b. This necessitates higher vertical zonal-wind shears in accordance with the thermal wind relationship.

\subsection{Vertical velocity and moisture distributions}

Moist processes are an important aspect of any GCM. In the aquaplanet simulations, the complex-physics parameterizations handle shallow and deep convection, cloud microphysics, cloud macrophysics, cloud-aerosol interactions, boundary-layer mixing, and surface fluxes. These processes are highly simplified or largely missing in the MITC simulation. However, the moist circulation patterns are comparable. In particular, we demonstrate that MITC compares particularly well to APS (NDC).

The time-mean zonal-mean CAM5-SE vertical pressure velocities, $\omega$, in the tropics are shown for (left) MITC, (middle) APS (NDC), and (right) APS in Fig. 2a-c. The overall shapes of the vertical pressure velocities are comparable. All simulations show a narrow updraft area, the upward branch of the Hadley circulation, close to the Equator and sinking motion poleward of about $7^{\circ} \mathrm{N} / \mathrm{S}$. However, Fig. $2 \mathrm{a}$ and $\mathrm{b}$ displays that the absence of the deep convection parameterization in MITC and APS (NDC) enhances the updraft speeds, narrows the updraft areas, and anchors the peaks in the lower atmosphere near $800 \mathrm{hPa}$. This is consistent with the notion that the vertical transport of the moist air into the upper troposphere is less effective without deep convection. Therefore, saturation is predominantly reached at lower levels and mainly removed by resolved-scale precipitation, which releases latent heat in the lower atmosphere and enforces the low-lying updrafts.

Furthermore, $\omega$ shows two equatorial updraft peaks in the APS simulation, one in the lower atmosphere near $800 \mathrm{hPa}$ and one in the upper atmosphere near $400 \mathrm{hPa}$. This suggests that the precipitation from the deep convection scheme provides enhanced heating at upper levels, which is also confirmed by the APS physics temperature forcing shown in Fig. 3b. The deep convection scheme thereby enhances the updrafts aloft and widens the updraft area in the tropics. Polewards of the tropical area the general circulation continues with a Ferrell and polar cell over the mid- and high latitudes, which leads to almost identical updrafts at around $60^{\circ} \mathrm{N} / \mathrm{S}$ and sinking motion over the polar regions (not shown).

MITC uses simple surface fluxes and boundary-layer diffusion to inject and mix moisture in the lower atmosphere, which is then transported and modulated globally by the resolved-scale flow and the moist physics parameterizations. Figure $2 \mathrm{~d}-\mathrm{f}$ shows the latitude-pressure cross sections of the time-mean zonal-mean-specific humidity in all three simulations. The general shapes and magnitudes of the specific humidity fields are largely similar. Differences are mostly apparent in the tropics at lower levels. Below $800 \mathrm{hPa}$ the aquaplanet simulations show extended areas with high moisture contents between $20^{\circ} \mathrm{N} / \mathrm{S}$, whereas the MITC simulation exhibits a narrower upward peak at the Equator.

The general latitudinal distributions of the surface latent heat fluxes in MITC and the aquaplanet simulations are comparable (not shown), although there is about $20 \%$ less surface latent heat release in MITC with a time-mean globalmean of $73 \mathrm{~W} \mathrm{~m}^{-2}$ vs. $93 \mathrm{~W} \mathrm{~m}^{-2}$ in APS. This is a contributor to the dryer lower atmosphere in MITC. However, the main cause for the difference in the low-level moisture distributions in Fig. $2 \mathrm{~d}-\mathrm{f}$ is that the MITC parameterizations do not mix the lower-atmosphere moisture as effectively as the complex aquaplanet parameterizations, which is an expected feature. This is confirmed by the total specific humidity tendencies in Fig. $3 c$ and d, which are recorded by the physical parameterization packages. In MITC (Fig. 3c), the positive specific humidity tendencies are solely caused by the boundary-layer mixing. The MITC mixing is strongest below $900 \mathrm{hPa}$ and quickly diminishes in the upward direction. In APS (Fig. 3d), the positive specific humidity tendencies in the tropics express the balance between the boundary-layer forcing and the moist-physics forcing (individual plots not shown). Here it is apparent that the resulting positive moisture tendencies in the lower APS troposphere (Fig. 3d) reach higher locations than their MITC counterparts (Fig. 3c). The positive aquaplanet moisture forcing maximizes at $800 \mathrm{hPa}$ between 10 and $20^{\circ} \mathrm{N} / \mathrm{S}$. This widens the areas with high specific humidity values in the aquaplanet simulations in comparison to the narrow MITC peak at the Equator.

As an aside, the deep convection parameterization in APS (Fig. 2f) transports equatorial moisture higher into the atmosphere than the APS (NDC) (Fig. 2e) or MITC (Fig. 2d), which is also apparent in the enhanced APS relative humidity values at the Equator in Fig. 2i. We also note that the moist physics parameterizations in the aquaplanet simulations allow for re-evaporation of precipitation as it falls, which enhances the specific humidity contents below precipitating clouds. Re-evaporation is not included in the MITC physics package. However, we tested a version with re-evaporation and the resulting moisture distribution is almost identical to the results shown here. Therefore, we do not pursue the reevaporation variant of the MITC design any further.

The time-mean zonal-mean relative humidity distributions are shown in Fig. $2 \mathrm{~g}-\mathrm{i}$, combining the information about the specific humidity and the general circulation of the atmosphere. Overall, all relative humidity patterns and magnitudes are similar. Some differences are visible in the polar regions in the mid-troposphere where the aquaplanet simulations exhibit higher relative humidity values. In addition, the MITC distribution shows somewhat larger dry cells in the subtropical downward branch of the Hadley circulation 

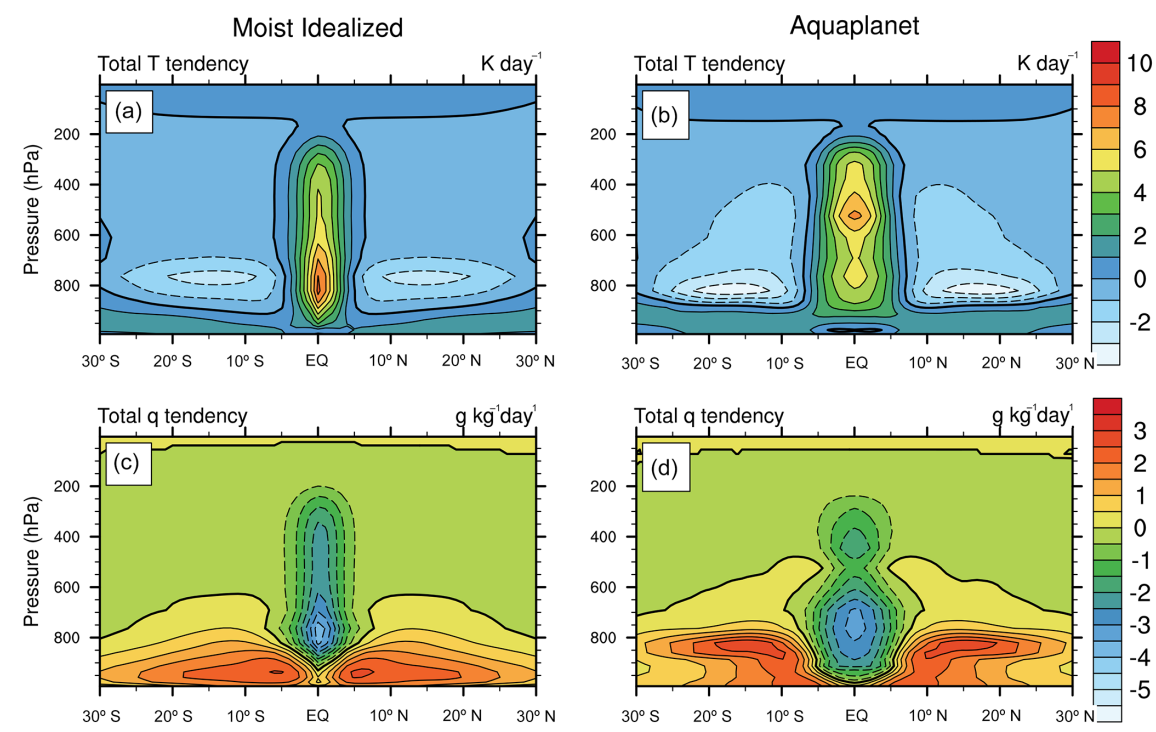

Figure 3. Comparison of CAM5-SE latitude-pressure cross sections in MITC (left column) and APS (right column): Time-mean zonal-mean $(\mathbf{a}, \mathbf{b})$ total temperature tendency and $(\mathbf{c}, \mathbf{d})$ total specific humidity tendency from the physical parameterization packages. The latitude range is $30^{\circ} \mathrm{S}$ to $30^{\circ} \mathrm{N}$.

(around $20^{\circ} \mathrm{N} / \mathrm{S}$ ) where precipitation is at a minimum as later shown in Fig. 4. The APS (NDC) and MITC configurations are less efficient at bringing moisture into the upper troposphere near the Equator, and tend to have higher relative humidity values throughout the lower troposphere in comparison to APS. In all three simulations, the relative humidity shows dry areas above the tropopause.

The characteristics of the time-mean zonal-mean total temperature tendencies and total specific humidity tendencies (Fig. 3a-d) have largely been mentioned in the discussions above. Here, we briefly highlight the close resemblance between the net physical forcing patterns and their magnitudes in the MITC and APS simulations. This cannot be taken for granted and is a result of the MITC parameter tuning, Here, we do not display the results for the APS (NDC) configuration because they closely resemble APS. The main difference between APS and APS (NDC) is the position of the condensational heating maximum in Fig. 3b, which moves downward in APS (NDC).

\subsection{Precipitation rates}

Figure 4 displays the CAM5-SE time-mean zonal-mean and hemispherically averaged precipitation rates for MITC in comparison to the (a) APS (NDC) and (b) APS simulations. The aquaplanet total precipitation rates (PRECT) are divided into large-scale precipitation (PRECL) and convective precipitation (PRECC). This distinction is important because MITC does not have a convection parameterization. Therefore, all of its total precipitation occurs at the grid scale (PRECT =PRECL). The overall distributions of the precipitation rates match qualitatively. The maximum precipita- tion rates are recorded at the Equator, the minima are located around $15-20^{\circ} \mathrm{N} / \mathrm{S}$, secondary midlatitudinal maxima are found between 35 and $40^{\circ} \mathrm{N} / \mathrm{S}$ and the precipitation diminishes at higher latitudes. All CAM5-SE simulations exhibit a singe precipitation peak in the tropics. MITC has less total equatorial precipitation than APS (NDC) (Fig. 4a), although the total moist idealized precipitation rate (in black) is nearly identical to the large-scale component of APS (NDC) denoted in blue. The additional precipitation (PRECC, in green) in APS (NDC) comes from the shallow convection scheme. Once deep convection is included (see Fig. 4b), the convective component of the equatorial precipitation PRECC increases and the large-scale component PRECL greatly decreases, while the equatorial peak widens to include a slightly larger range of latitudes. However, the total precipitation rate in APS is comparable to the precipitation rate in MITC, and their equatorial peaks overlay each other.

The precipitation rates in Fig. 4 are consistent with Table 2 that lists the global-mean time-mean precipitation rates and the time-mean zonal-mean peak precipitation rates at the Equator. Note that the time-mean globally averaged total APS precipitation rate in Table 2 is much larger $\left(3.21 \mathrm{mmday}^{-1}\right)$ than the values for MITC because it includes the convective precipitation. The globally averaged large-scale precipitation rate for APS (NDC), $2.33 \mathrm{~mm}$ day $^{-1}$, is quite comparable to MITC, $2.10 \mathrm{~mm} \mathrm{day}^{-1}$. As mentioned above, the equatorial peak rates in MITC are also quite comparable to the large-scale PRECL rate in APS (NDC). Overall, MITC has less globalmean precipitation than APS, which is also true when compared to reanalysis data (around $3 \mathrm{~mm} \mathrm{day}^{-1}$, see e.g., Saha et al., 2010). This could be changed when, for example, in- 
Table 2. Time-mean precipitation statistics for CAM5-SE in units mm day ${ }^{-1}$ : global-mean and zonal-mean at the Equator showing the total precipitation rates (PRECT) and the fraction of large-scale (PRECL) vs. convective (PRECC) precipitation rates for MITC, APS NDC and APS.

\begin{tabular}{lllrr}
\hline $\begin{array}{l}\text { Model } \\
\text { configuration }\end{array}$ & Acronym & $\begin{array}{l}\text { Precipitation } \\
\text { type }\end{array}$ & $\begin{array}{r}\text { Global-mean } \\
\text { precipitation }\end{array}$ & $\begin{array}{r}\text { Equatorial } \\
\text { precipitation }\end{array}$ \\
\hline Moist idealized & MITC & Total & 2.10 & 19.68 \\
Aquaplanet (no deep) & APS NDC & Total & 3.24 & 27.68 \\
& & Large-scale & 2.33 & 19.35 \\
& & Convective & 0.91 & 8.33 \\
Aquaplanet & APS & Total & 3.21 & 17.97 \\
& & Large-scale & 1.18 & 4.88 \\
& & Convective & 2.03 & 13.09 \\
\hline
\end{tabular}
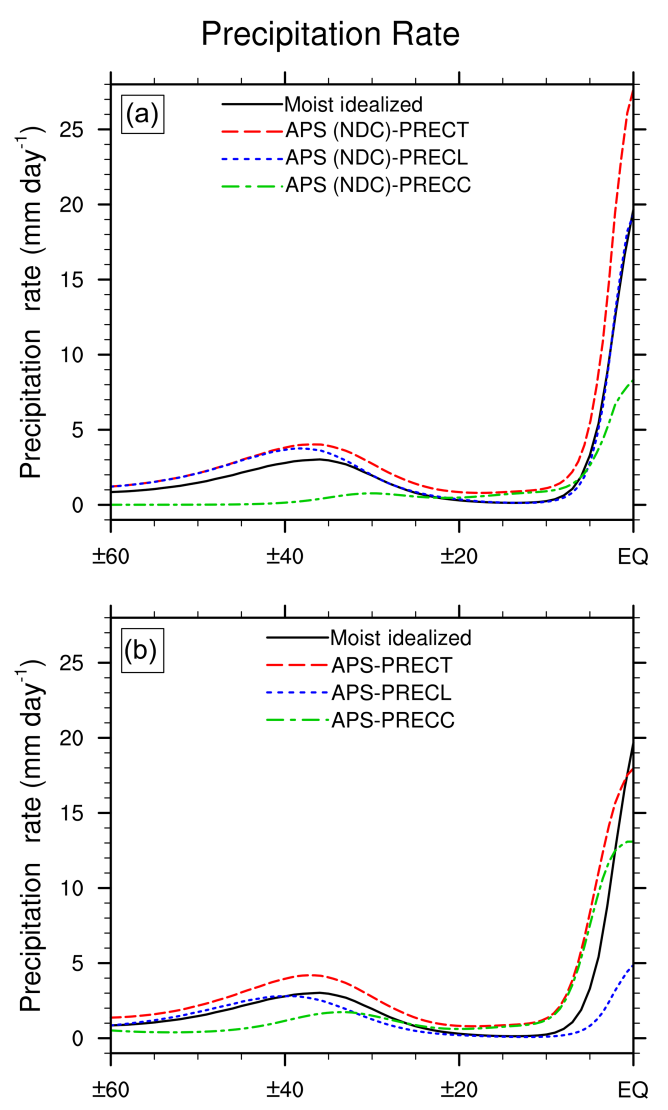

Figure 4. CAM5-SE time-mean zonal-mean precipitation rate (hemispherically averaged) for MITC (black) compared to (a) APS (NDC) and (b) APS. The total aquaplanet precipitation rate (PRECT, red dashed) is divided into the large-scale precipitation rate (PRECL, blue dotted) and convective precipitation rate (PRECC, green dash-dot).

creasing the bulk transfer coefficients in the current MITC surface flux parameterization. However, this should be done with care to guarantee that the global circulation is still reasonable as demonstrated via the current MITC parameter choices. In addition, we emphasize that a perfect match be- tween MITC and aquaplanet simulations cannot be expected and is not the goal of our study. Our goal is to create a reasonable match.

Figure 5 shows the CAM5-SE frequency distribution of the precipitation rates in MITC and APS, where APS splits up the contributions from the total, large-scale, and convective (PRECT, PRECL, PRECC) precipitation rates. In particular, the figure displays the fraction of the precipitation rate between $10^{\circ} \mathrm{N}$ and $10^{\circ} \mathrm{S}$ that falls into each precipitation bin. This analysis is based on 6 months of 6-hourly instantaneous data (no time averaging). The data have been conservatively regridded to a $2^{\circ} \times 2^{\circ}$ latitude-longitude grid before the analysis, as recommended by Chen and Knutson (2008) and Williamson (2008a). In particular, Williamson (2008a) argued that precipitation extremes should be analyzed on spatial scales that are larger than(ideally double) the size of the truncation limit of the model. This lessens the impact of somewhat different grid sizes when intercomparing different models. In addition, it leads to a more robust analysis because precipitation extremes are not modeled reliably at the grid scale and often do not converge with increasing horizontal resolution. The precipitation rates in Fig. 5a range from 0 to $120 \mathrm{~mm} \mathrm{day}^{-1}$ with $1 \mathrm{mmday}^{-1}$ bins, and Fig. $5 \mathrm{~b}$ ranges from 0 to $600 \mathrm{~mm} \mathrm{day}^{-1}$ with $10 \mathrm{~mm} \mathrm{day}^{-1}$ bins.

The presence of the convective precipitation in the aquaplanet simulation leads to different precipitation frequency distributions in Fig. 5. MITC has a greater fraction of its precipitation occurring at rates over $40 \mathrm{mmday}^{-1}$. This is an expected result because MITC has no sub-grid parameterizations and requires saturation on the grid scale to initiate precipitation. This allows the moisture to build up and the resulting rainfall occurs in large events with greater precipitation rates, as seen in Fig. 5b. Often, the extreme events can be characterized as isolated "grid-point storms". Below $40 \mathrm{~mm} \mathrm{day}^{-1}$ the APS shows a greater fraction of precipitation rate. As seen by the green line, this lighter precipitation is mostly triggered by the convective parameterizations, which includes both the shallow and deep convection schemes. These analysis results change significantly when MITC is compared to the aquaplanet simulation without the 

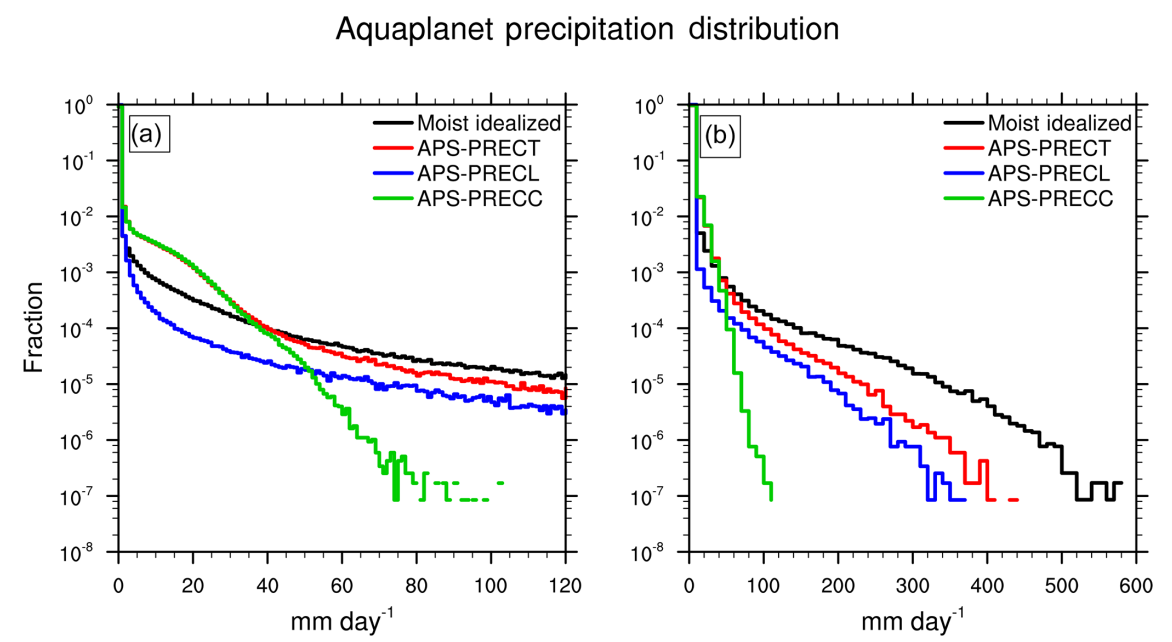

Figure 5. Precipitation frequency distribution of the SE precipitation rates averaged between $\pm 10^{\circ}$ for MITC (black), aquaplanet total precipitation (PRECT, red), large-scale precipitation (PRECL, blue), and convective precipitation (PRECC, green) ranging from (a) 0 to $120 \mathrm{~mm} \mathrm{day}^{-1}$ with $1 \mathrm{~mm} \mathrm{day}^{-1}$ bins and (b) 0 to $600 \mathrm{~mm} \mathrm{day}^{-1}$ with $10 \mathrm{~mm} \mathrm{day}^{-1}$ bins.

Aquaplanet (no deep conv.) precipitation distribution
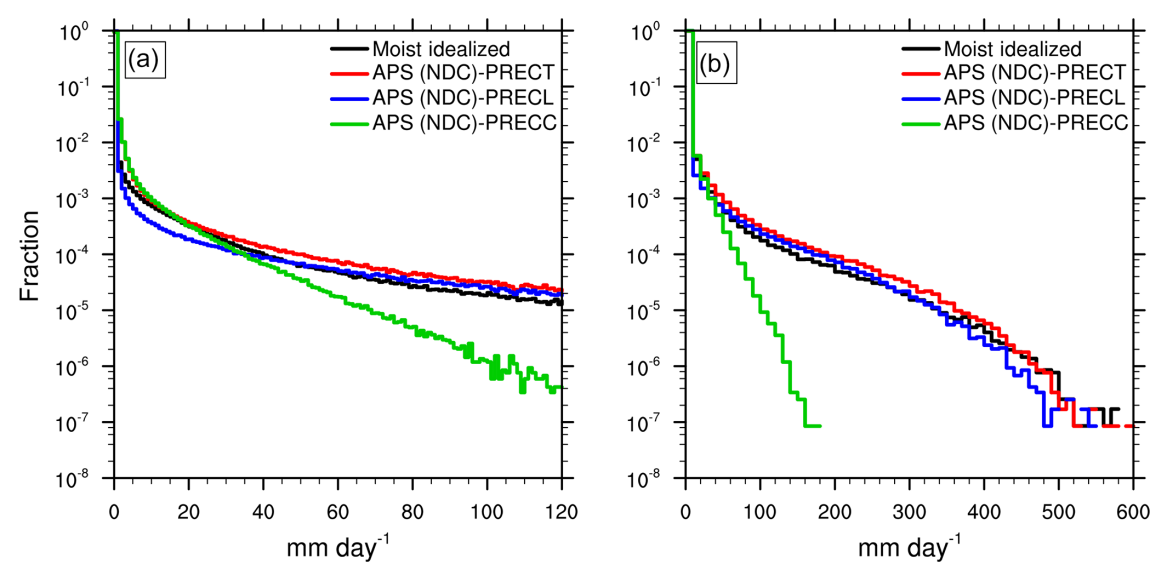

Figure 6. Same as Fig. 5 except comparing to APS (NDC).

deep convection scheme as showcased in Fig. 6. Here, APS (NDC) has a total precipitation rate distribution (in red) that lies on top of the MITC distribution. This impressive match suggests that the MITC configuration produces an appropriate precipitation rate distribution for a GCM without a deep convection parameterization.

\subsection{Convectively coupled equatorial waves}

As the last point of comparison, we analyze the convectively coupled equatorial waves in all three CAM5-SE configurations with MITC, APS (NDC), and APS at the resolution of $110 \mathrm{~km}$. Figure 7 shows the wavenumber-frequency spectra for all simulations. These plots display the spectral power as a function of wavenumber and frequency and are generated using 96-day windows with 60 days of overlap, as described by Wheeler and Kiladis (1999). The analysis is based on 6 months of 6-hourly instantaneous data between $15^{\circ} \mathrm{S}$ and $15^{\circ} \mathrm{N}$. We select the temperature at $100 \mathrm{hPa}$ instead of the often used outgoing longwave radiation (OLR), because the latter is not available in the moist idealized physics simulation. The solid lines are dispersion curves that indicate lines of constant equivalent depth with $h=12,25$, and $50 \mathrm{~m}$. They are derived from shallow-water theory and give information about the vertical wave lengths and phase speeds. In particular, the relation between the phase speed $c_{\mathrm{ph}}$ and the equivalent depth is $c_{\mathrm{ph}}=\sqrt{\mathrm{gh}}$ in the absence of a zonal background flow. The thick dashed line is the $h=200 \mathrm{~m}$ dispersion curve. 


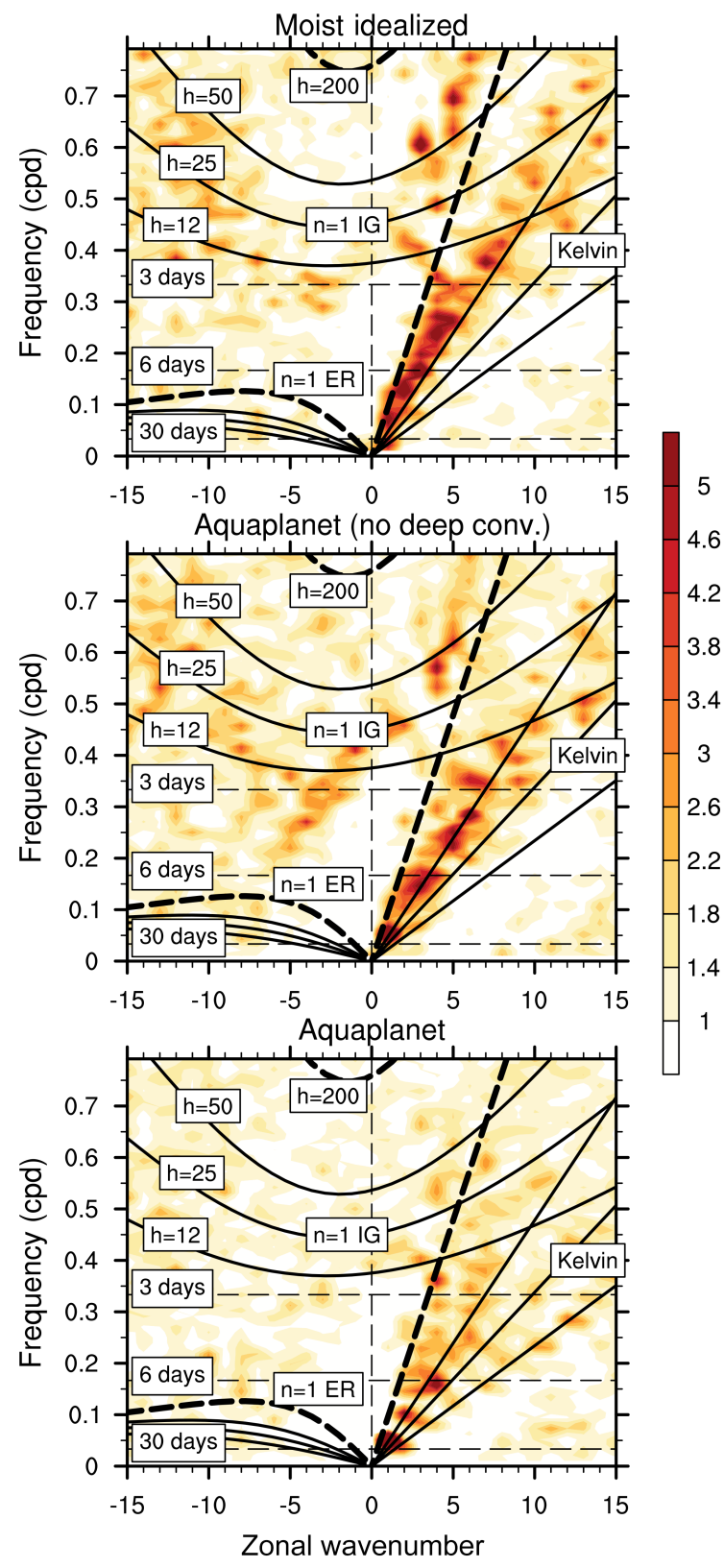

Figure 7. Wavenumber-frequency diagrams for CAM5-SE showing spectral power for the symmetric component of the $100 \mathrm{hPa}$ equatorial temperature averaged between $\pm 15^{\circ}$ in the (a) MITC, (b) APS (NDC), and (c) APS simulations. The solid lines show the theoretical shallow-water dispersion curves for the equivalent depths $h=12,25$, and $50 \mathrm{~m}$ without a mean background velocity. The thick dashed line is the $h=200 \mathrm{~m}$ dispersion curve.

Figure 7 displays the symmetric components of the equatorial waves after the background spectrum has been removed following the Wheeler and Kiladis (1999) approach. The asymmetric components of the wavenumber-frequency spectra are not shown because they do not exhibit any statistically significant wave activity. Figure 7 includes disper- sion curves for eastward traveling Kelvin waves, eastward and westward $n=1$ inertio-gravity waves (IG), and westward traveling equatorial $n=1$ Rossby waves (ER) where $n$ denotes the mode number. MITC features Kelvin waves for equivalent depth of over $h=50 \mathrm{~m}$ with periods ranging mainly from 3 to 30 days. APS also contains Kelvin waves, although they have an equivalent depth of about $h=50 \mathrm{~m}$ and are strongest at wavenumber 1 with a period of 20 30 days. The APS (NDC) contains similar, slightly more intense than APS, Kelvin waves, with dominant periods between 3 and 30 days.

As the convection parameterization simplifies, from shallow and deep convection in APS to no parameterizations in MITC, the strengths and the phase speeds of the Kelvin waves tend to increase. The increased phase speeds are equivalent to larger equivalent depths according to the dispersion relation quoted above. The Kelvin waves in MITC seem to be more prevalent at higher frequencies (shorter wave periods). These changes are linked to the varying complexity of the precipitation processes that act as the wave generator. In general, the precipitating systems in the tropics are more organized in APS whereas the precipitation regimes are "spottier" in APS (NDC) and MITC with more grid-pointlike storms. The latter is an expected characteristic of models without a deep convection parameterization. This characteristic is also visible in Hovmöller diagrams of instantaneous tropical precipitation rates (not shown) and indirectly in the $850 \mathrm{hPa}$ vertical pressure velocities in Fig. 8, which are discussed in the next section.

Both MITC and APS (NDC) also feature Kelvin waves with very high frequencies, greater than 0.5 cycles per day ( 2 day waves), and very large equivalent depth $(h \approx$ $200 \mathrm{~m}$ ). This $200 \mathrm{~m}$ equivalent depth is the theoretically determined depth associated with the peak projection response to deep convective heating, as described by Wheeler and Kiladis (1999). These "nonconvectively coupled" waves have a half-wavelength of about $14 \mathrm{~km}$, therefore extending over the depth of the tropical troposphere. These waves are very common in dynamical fields, but are typically missing in Wheeler-Kiladis diagrams based on OLR data. For comparison, some examples of the CAM-SE (also called "HOMME") aquaplanet wavenumber-frequency spectra based on OLR data are presented in Mishra et al. (2011a), who highlighted the convectively coupled waves with typical equivalent depths between 12 and $50 \mathrm{~m}$. In addition, Semane and Bechtold (2015) provide additional pointers for comparisons and showed symmetric OLR wavenumber-frequency spectra from aquaplanet simulations at a T159 spectral transform resolution with a linear Gaussian grid $(\approx 125 \mathrm{~km}$ grid spacing). In particular, they showed results without and with a deep convection scheme, which suggest that the inclusion of their convection scheme leads to slightly more energetic wave spectra. However, this finding is different from our result in Fig. 7 that show enhanced Kelvin wave activity without the deep convection parameter- 


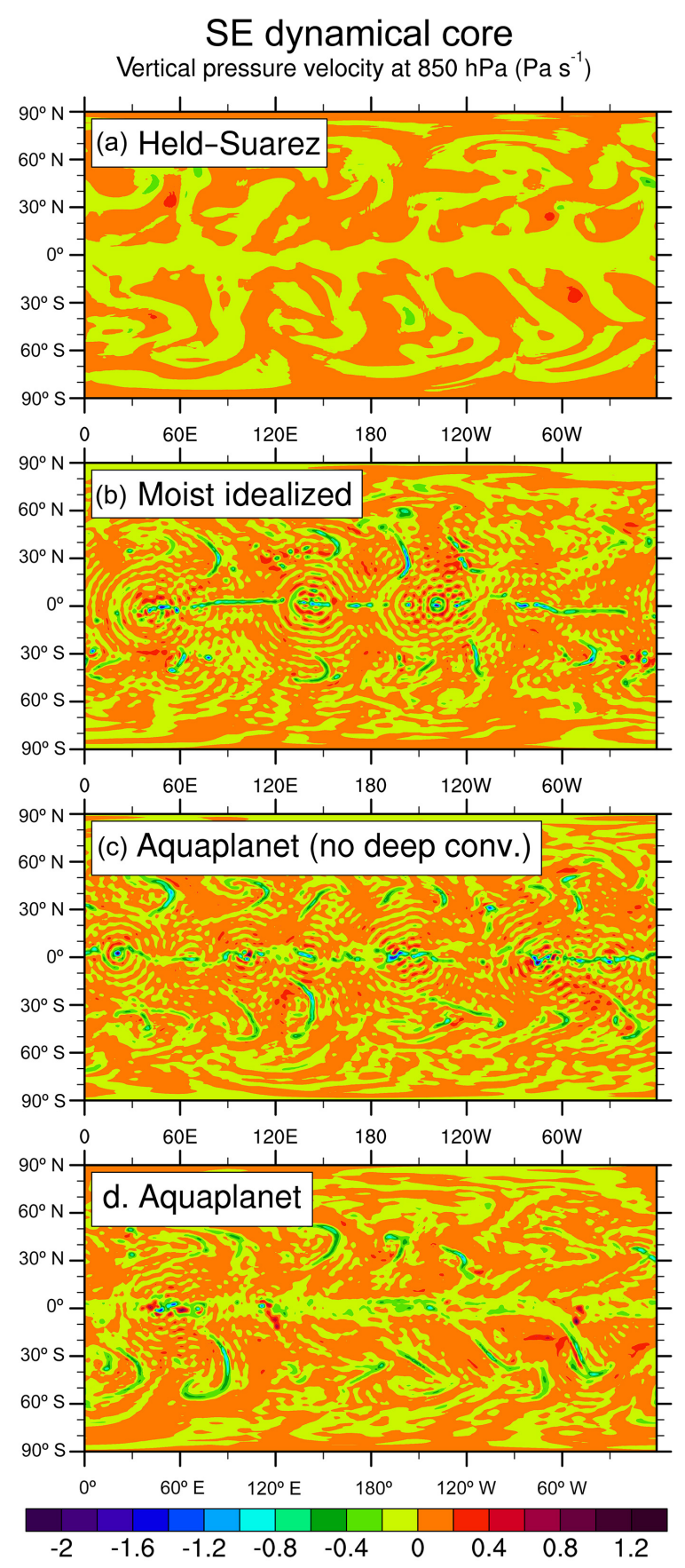

Figure 8. Instantaneous latitude-longitude snapshots of the $850 \mathrm{hPa}$ vertical pressure velocity in CAM5-SE for the (a) dry Held-Suarez test case, (b) MITC, (c) APS (NDC), and (d) APS.

ization. These differences could be related to the nature of the convection parameterizations in both models and the fact that Fig. 7 is based on a different ( $100 \mathrm{hPa}$ temperature) data set. Such aspects will require further investigation.

\section{MITC example applications}

The analysis in Sect. 4 demonstrates that the overall climatic states in MITC and the aquaplanet simulations are comparable. Despite its simplicity, the moist idealized simulation generates convectively coupled equatorial waves and reasonable precipitation distributions. Furthermore, the characteristics of the general circulation and the precipitation-related processes in the moist idealized test case makes convincing arguments that the circulation is an adequate candidate for applications, such as the analysis of the physics-dynamics coupling or a dynamical core intercomparison. Snapshots of these example applications are presented in the next two subsections.

\subsection{An analysis of the physics-dynamics coupling in CAM5-SE}

We now demonstrate the strength of the MITC approach in revealing the intricacies of the physics-dynamics coupling strategy in CAM5-SE. This aspect cannot be investigated in dry dynamical core tests, and physics-dynamics coupling questions have recently received renewed interest as documented by Gross et al. (2016). Figure 8 shows instantaneous, randomly selected latitude-longitude snapshots of the $850 \mathrm{hPa}$ vertical pressure velocity from (a) dry HS, (b) MITC, (c) APS (NDC), and (d) APS simulations. These snapshots were taken after the simulations had fully spun up. The dry HS snapshot of SE in Fig. 8a exhibits the typical structure of updrafts along the Equator, which mimic the Intertropical Convergence Zone (ITCZ). In addition, the updrafts and downdrafts in the midlatitudes are aligned with the frontal zones of the baroclinic systems. The contours in the dry HS simulation are very smooth with occasional spectral ringing signatures at higher latitudes. However, in the moist MITC simulation with SE (Fig. 8b) distinct, large-scale ringing patterns are visible. They originate from grid-point-scale areas of precipitation along the Equator as shown in Fig. 9c and $\mathrm{d}$. This gravity wave ringing is also visible in APS (NDC) in Fig. 8c and to a lesser degree in the APS experiment with SE (Fig. 8d) where the circular patterns transform into many broken and noisy contours. However, some broken gravity wave circles near the Equator are still visible around $50^{\circ} \mathrm{E}$ in APS.

This suggests that the deep convection parameterization reduces the occurrences of the grid-scale precipitation events and thereby the large-scale gravity wave generation in APS. This is a desired and expected outcome because the deep convection parameterization removes moisture before the grid box reaches saturation and thereby prevents large amounts of condensed water from being removed in a single time step. However, the CAM5 deep convection parameterization cannot eliminate the overall very noisy and undesirable flow characteristics. We conclude that small areas with extreme precipitation enhance the gravity wave activity in CAM5-SE, 


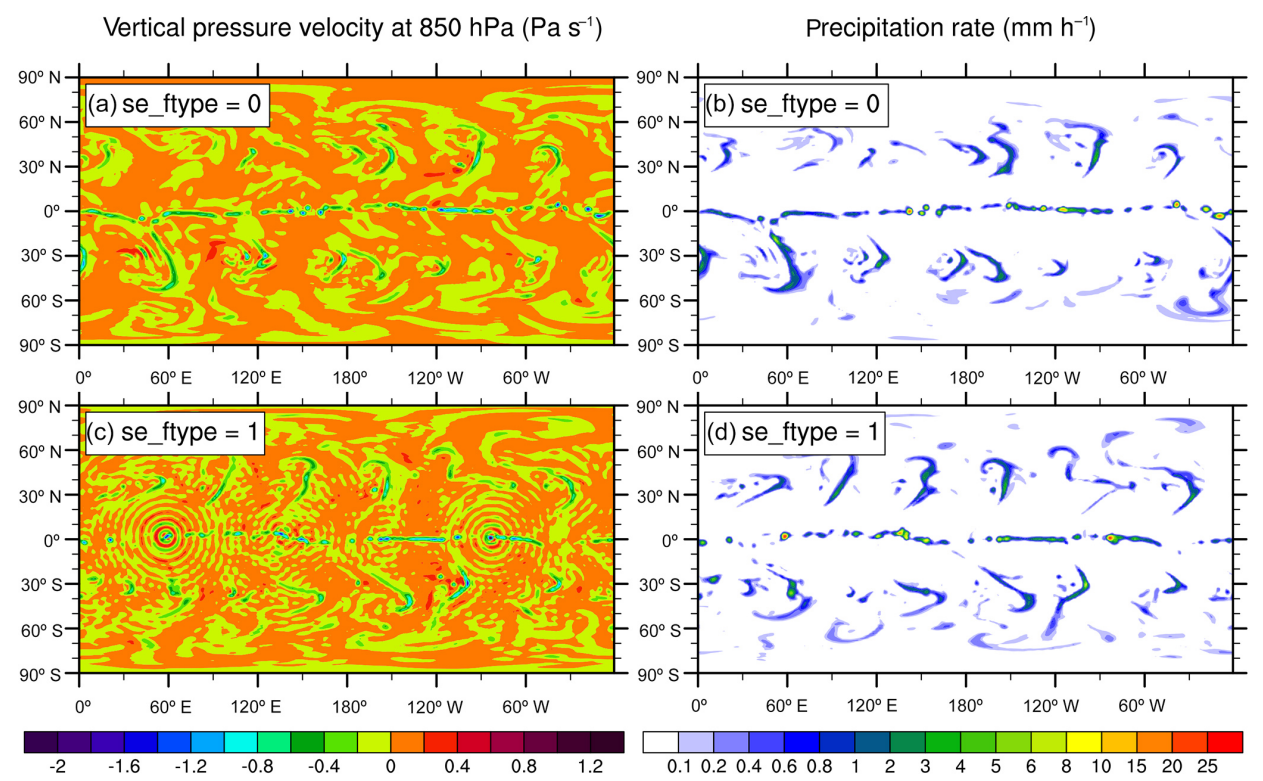

Figure 9. Instantaneous latitude-longitude snapshots of the (left) $850 \mathrm{hPa}$ vertical pressure velocity and (right) large-scale precipitation rate for two different physics-dynamics coupling strategies in CAM5-SE with moist idealized physics (MITC). (a, b) gradual application of the physics tendencies in the sub-cycled dynamical core $\left(s_{-}\right.$ftype $\left.=0\right)$ and $(\mathbf{c}, \mathbf{d})$ sudden adjustment of the prognostic variables at each physics time step (se_ftype $=1$ ).

but that they are not the sole cause of the noise. The noise must be linked to a different mechanism, and the MITC approach now serves as an idealized test bed to distinguish between causes and effects. Because the phenomenon has been isolated in MITC, only very few mechanisms and model variants need to be explored instead of the many choices in APS.

As mentioned above, Fig. $9 \mathrm{c}$ and d reveal that the centers of the gravity wave ringing patterns in MITC are co-located with intense grid-point storms, which are located along the Equator near $60^{\circ} \mathrm{E}$ and $90^{\circ} \mathrm{W}$ in this random snapshot. In Fig. 8a it was also revealed that the ringing is absent if the dynamical core is coupled to the dry Held-Suarez physics. This strongly suggests that the source of the noise does not lie within the dynamical core, but either comes from the moist physics processes or originates at the coupling interface between the dynamical core and the physics. The latter aspect is indeed the source of the problem. As mentioned in Sects. 2.6 and 3, our version of CAM5-SE utilized the se_ftype $=1$ coupling strategy and therefore the state variables are suddenly adjusted at the end of the relatively long physics time step (30 $\mathrm{min}$ ) before the dynamical core is called again. This also incorporates the sudden adjustment of the pressure field in the case that moisture was removed or added in a grid column by the physical parameterizations.

An alternative coupling strategy is provided in CAM5-SE as an input option (se_ftype $=0$ ) that transfers the forcing tendencies from the physics parameterization package to the dynamical core. The dynamical core then applies the physical forcing tendencies gradually with the sub-cycled, and thereby short $(5 \mathrm{~min})$, dynamics time step. The results of this MITC simulation are depicted in Fig. 9a and b. Nothing else was changed. As before, the figure shows an instantaneous, randomly selected snapshot of the (left) $850 \mathrm{hPa}$ vertical pressure velocity field and (right) the precipitation rate. Strong grid-scale precipitation areas are still present along the Equator, but the circular gravity wave patterns are eliminated. This simulation now has very similar flow characteristics to the dry HS simulation, but with enhanced updraft and downdraft speeds as expected in the more energetic moist simulation. There are occasional spectral ringing patterns, but overall the contours are very smooth. The more gradual se_ftype $=0$ coupling choice has therefore been adopted as the default in the most recent versions of CAM5-SE.

As an aside, we had also analyzed the impact of other modeling choices on the gravity wave noise in the CAM5SE se_ftype $=1$ configuration. These were variations of the hyper-diffusion coefficients and the switch from the floating Lagrangian vertical coordinate with periodic remapping (default) to a finite-difference treatment of the vertical derivatives in the dynamical core. None of these dynamical core adjustments made a substantial difference and are therefore not shown. This confirmed our original hypothesis that the gravity wave ringing did not originate from the dynamical core, but rather from its interaction with the moist physical parameterizations. 


\subsection{Snapshots of a moist dynamical core intercomparison}

The preceding subsection highlights why simplified moist dynamical core test cases like MITC are helpful in the development and testing of GCMs. Furthermore, the MITC approach can also be utilized to intercompare different dynamical cores, which we present here as a second application example. We now focus on the characteristics of all four CAM5 dynamical cores (SE, FV, EUL, and SLD), which are available as options in NCAR's CESM modeling framework. Each dynamical core uses the identical implementation of the MITC physics package. In addition, selected snapshots, such as those comparing the kinetic energy spectra or precipitation distributions in MITC and APS, use the same CAM5 complex-physics package with identical tuning coefficients for APS. Therefore, any differences in the results are due to the different numerical designs of the dynamical cores and their physics-dynamics coupling interfaces. This reveals the impact of the dynamical cores on the general circulation and is a contribution to the very few systematic intercomparisons of moist dynamical cores.

Besides the tropical cyclones studies by RJ12 and Reed et al. (2015), the only systematic long-term "climate" assessments were presented by Williamson (2008b). He conducted an aquaplanet comparison of FV and EUL with the predecessor CAM3 physics package and also investigated the impact of increasing horizontal resolutions on the climate statistics. Other intercomparisons, such as the international Aqua-Planet Experiment (Williamson et al., 2012; Blackburn et al., 2013), the Atmospheric Model Intercomparison Project (AMIP; see Gates et al., 1999), or the Intergovernmental Panel for Climate Change (IPCC) assessments, are conducted with different dynamical cores and different physics packages, making it impossible to distinguish between causes and effects.

As described in Sect. 3, the CAM5 dynamical cores use different numerical methods and grid designs, and therefore represent a variety of choices commonly used in GCMs. No special dynamical core tuning is applied and each dynamical core uses its default settings, such as the time step length and diffusion coefficients for the approximate $1^{\circ}$ resolutions (see Table 1). For brevity, we only present selected snapshots of the MITC intercomparison, including the assessment of numerical noise, the comparison of the diffusive properties via the kinetic energy spectra, as well as rainfall and tropical wave assessments.

\subsubsection{An assessment of numerical noise}

In order to connect our discussion to Sect. 5.1, we first shed light on the presence of gravity wave noise in the other three dynamical cores when coupled to the MITC physics package. Instantaneous, randomly selected snapshots of their $850 \mathrm{hPa}$ vertical pressure velocities are shown in Fig. 10, which can

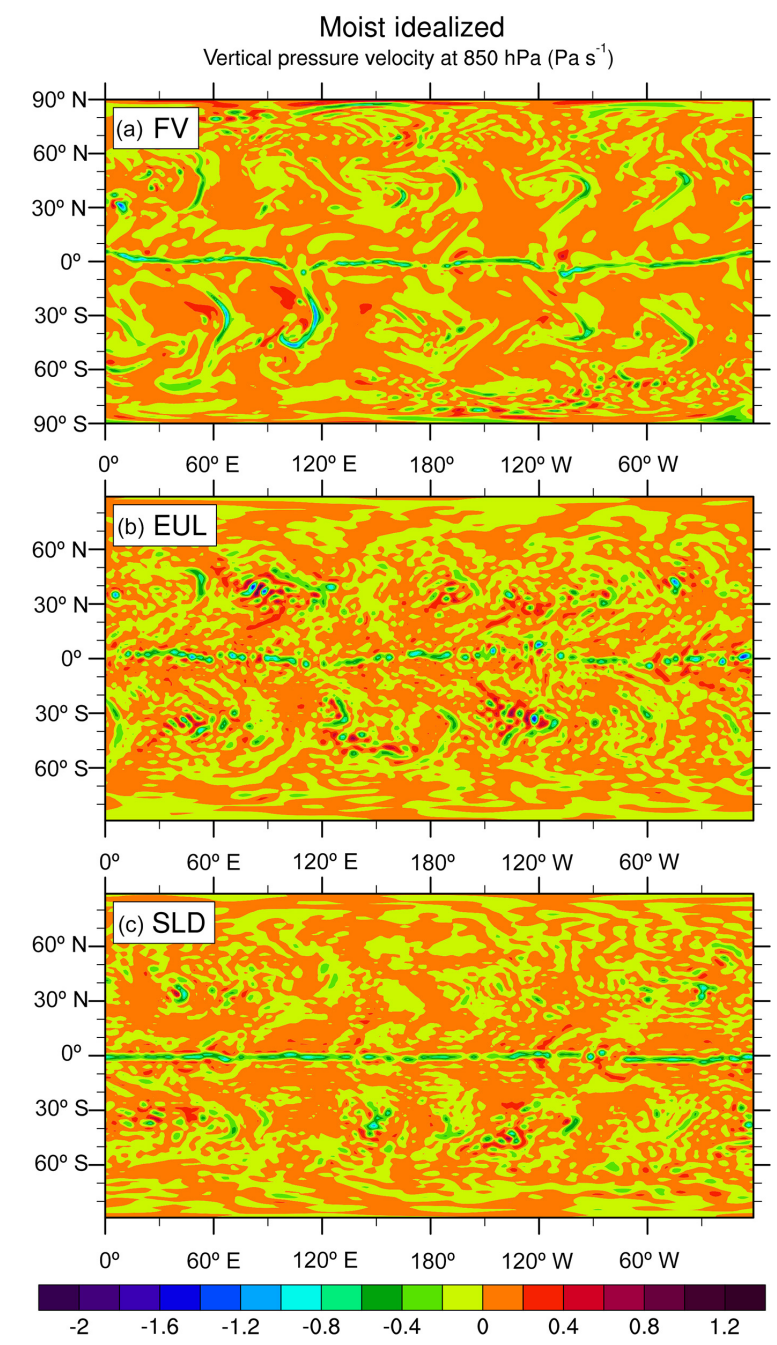

Figure 10. Instantaneous latitude-longitude snapshots of the $850 \mathrm{hPa}$ vertical pressure velocity in MITC simulations with (a) FV, (b) EUL, and (c) SLD.

readily be compared to the corresponding SE $850 \mathrm{hPa}$ vertical pressure velocities in Figs. 8b and 9a and c. Again, all models were fully spun up before the snapshots were taken, and the snapshots are representative of the whole simulation.

The snapshots show that the circular gravity wave signatures of the SE dynamical core are not present in the other three dynamical cores. This is despite the fact that FV, EUL, and SLD are also implemented with the sudden physics adjustment strategy at the end of the physics time step, and that they occasionally exhibit grid-point-size storms. The physics time steps (30 min) are identical in SE, FV, and SLD while a shorter physics time step $(10 \mathrm{~min})$ is required in EUL for numerical stability reasons. 
Despite the short EUL physics time step, which should have helped lessen the impact of the sudden physical adjustments as seen in SE (Fig. 9), the EUL simulation in Fig. 10b is the noisiest of the three. The spectral ringing, the so-called Gibbs phenomenon, of the spectral transform method breaks up the structured systems and creates many checkerboardlike updraft and downdraft patterns. The Gibbs phenomenon is caused by the need to represent fields with discontinuities or sharp gradients by smooth global basis functions. Such noise in the vertical velocity field can cause spurious noisy rainfall, which is sometimes called "spectral rain". Increasing the strength of the explicitly added diffusion lessens the Gibbs ringing in EUL, as demonstrated by Jablonowski and Williamson (2011) in dry simulations, but might generally be undesirable and can degrade other climate diagnostics. The magnitudes of the updrafts and downdrafts in EUL are the most extreme in comparison to the other three dynamical cores, and the updraft maxima (around $-2.8 \mathrm{~Pa} \mathrm{~s}^{-1}$ ) exceed the chosen color scale. These updraft peaks are fueled by intense grid-scale-size precipitation events (not shown here), which have the strongest effect on EUL's vertical pressure velocities in the midlatitudes.

The vertical pressure velocity patterns in the SLD simulation (Fig. 10c) are also very noisy, but their magnitudes are less intense. SLD, like EUL, is based on the spectral transform method and is impacted by the same Gibbs phenomenon. The SLD simulation is somewhat more diffusive than EUL (see also Fig. 11 discussed next) due to the built-in semi-Lagrangian interpolations in the numerical scheme and the Gibbs ringing is more damped. In contrast, the FV simulation (Fig. 10a) shows rather smooth contours in the tropics and midlatitudes, highlighting the presence of the ITCZ and baroclinic systems. However, at high latitudes the contours are less organized and broken. This may be caused by the converging meridians of the latitude-longitude grid near the poles and the polar filtering needed for numerical stability. The MITC approach thereby allows for a systematic assessment of this aspect and the polar filter strength (not discussed here).

\subsubsection{Kinetic energy spectra}

The kinetic energy (KE) spectra provide insight into how atmospheric motions are distributed across spatial scales, and are used to assess the quality of the discretizations and their diffusive properties. Theory and observations suggest that the spectrum should have a slope of $k^{-3}$ where $k$ symbolizes the spherical wavenumber (Nastrom and Gage, 1985). Such a slope corresponds to the downscale cascade of enstrophy, while at small wavelengths (less than approximately $400 \mathrm{~km}$ ) the slope transitions to $k^{-5 / 3}$, corresponding to the downscale cascade of energy (Skamarock, 2011). The $k^{-5 / 3}$ regime cannot be presented in $1^{\circ}$ simulations, but is expected at higher horizontal resolutions $\left(0.25^{\circ}\right.$ and finer $)$ as shown by Jablonowski and Williamson (2011) (their Sect. 13.3.8) and

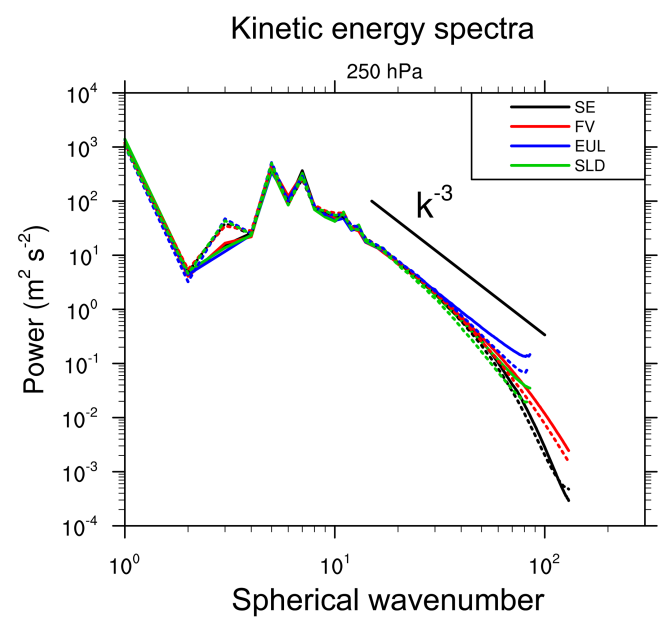

Figure 11. $250 \mathrm{hPa}$ kinetic energy spectra for MITC (solid lines) and APS (dashed lines) simulations with SE (black), FV (red), EUL (blue), and SLD (green). The slopes can be compared to the theoretical $k^{-3}$ slope.

Evans et al. (2013). In general, the downward curve in the kinetic energy at high wavenumbers near the truncation limit results as kinetic energy is removed via explicitly added and numerically implicit diffusion mechanisms.

Figure 11 shows the $250 \mathrm{hPa}$ kinetic energy spectra for the MITC simulations compared to APS for all four dynamical cores. The spectra for the APS (NDC) are very similar to APS and are therefore not shown. The spectra are cutoff near the highest resolved wavenumber based on the horizontal resolution. For SE and FV we select a cutoff of about $k=130$, while for EUL and SLD the highest resolved wavenumber is 85.

The aquaplanet and moist idealized kinetic energy spectra perfectly overlay each other in the low wavenumber regime. At higher wavenumbers, APS is slightly more diffusive than the MITC simulations, as depicted by the steeper slopes. However, the general characteristics of the four APS kinetic energy spectra is replicated in MITC. In particular, the two SE simulations show the steepest descents and are most diffusive near the truncation limit. These are followed by the pairs of the FV and SLD simulations whereas the two EUL slopes are the shallowest. The EUL spectra drop off in almost perfect accordance with the theoretical $k^{-3}$ slope.

Based on these slope assessments, one might conclude that EUL exhibits the highest "effective resolution", which Skamarock (2004) defines as the point where the drop-off of the $\mathrm{KE}$ spectra becomes steeper than the theoretical line. Therefore, EUL might be viewed as the most accurate dynamical core. However, this is clearly not the case when taking into account EUL's instantaneous vertical pressure velocity field (Fig. 10b). On the contrary, this analysis suggests that EUL's kinetic energy near the truncation limit is falsely elevated due to the presence of small-scale noise, and that models with 


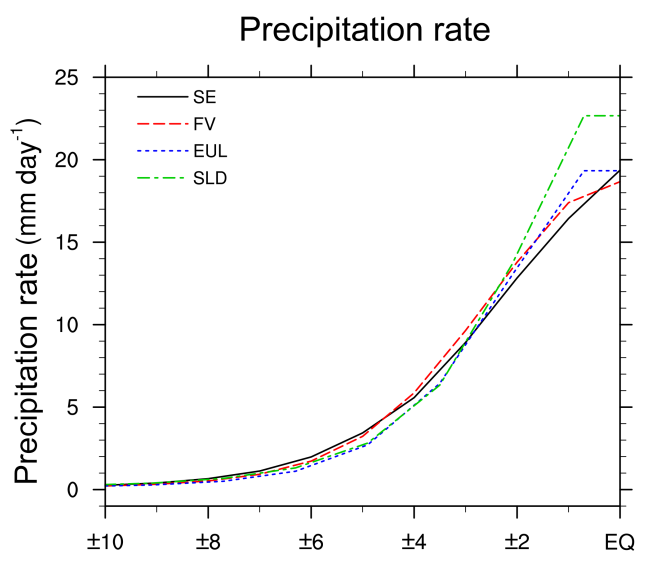

Figure 12. MITC time-mean zonal-mean hemispherically averaged precipitation rates for SE (black), FV (red dashed), EUL (blue dotted), and SLD (green dash-dot).

adequate diffusive properties near the grid scale are likely to exhibit steeper than $k^{-3}$ slopes at high wavenumbers. Of course, very steep drop-offs and early departures from the theoretical slope do indeed indicate that the explicitly added or implicit numerical diffusion impacts the larger scales. This reduces the dynamical core's ability to truthfully represent mid-scale to smaller-scale waves, which is undesirable.

In summary, the discussion demonstrates that the steepness of the slopes of the kinetic energy spectra are dominated by the dynamical cores. The KE distributions in the MITC simulations mimic the behavior of complex aquaplanet simulations, which mimic the behavior of realistic full-physics simulations with topography as shown by Evans et al. (2013). Therefore, MITC can be used as an idealized tool to assess, compare, and tune the diffusive properties of moist model configurations.

\subsubsection{Precipitation-related processes}

The next analyses focus on selected precipitation-related processes. The general characteristics of the time-mean, zonalmean precipitation rates of all four MITC dynamical cores are shown in Fig. 12. The forcing terms and zonal results are symmetric about the Equator, therefore the two hemispheres have been averaged together to reduce sampling variability. The precipitation rates are similar, especially for the precipitation rates in the midlatitudes and polar regions (not shown), which all overlay the MITC SE precipitation rate depicted in Fig. 4. However, the MITC precipitation rates in the tropics differ somewhat as displayed in Fig. 12 and Table 3. SLD has the highest tropical precipitation rate between $\pm 3-0^{\circ}$, with an equatorial peak of about $23 \mathrm{~mm} \mathrm{day}^{-1}$, followed by EUL, $\mathrm{FV}$, and SE in descending order. The EUL, FV, and SE simulations have very similar peak precipitation rates between 18.6-19.7 mm day $^{-1}$ at the Equator, but are characterized by slightly different latitudinal distributions. The two spectral
Table 3. Comparison of the time-mean global-mean total precipitation rates (PRECT) and the time-mean zonal-mean precipitation rates closest to the Equator in units mm day ${ }^{-1}$. All four CAM5 dynamical cores are listed in MITC and APS mode.

\begin{tabular}{llrr}
\hline $\begin{array}{l}\text { Model } \\
\text { configuration }\end{array}$ & $\begin{array}{l}\text { Dynamical } \\
\text { core }\end{array}$ & $\begin{array}{r}\text { Global-mean } \\
\text { precipitation }\end{array}$ & $\begin{array}{r}\text { Equatorial } \\
\text { precipitation }\end{array}$ \\
\hline Moist idealized (MITC) & SE & 2.10 & 19.68 \\
& FV & 2.10 & 18.62 \\
& EUL & 2.18 & 19.54 \\
& SLD & 2.27 & 22.76 \\
\hline Aquaplanet (APS) & SE & 3.21 & 17.97 \\
& FV & 3.23 & 20.03 \\
& EUL & 3.17 & 19.19 \\
& SLD & 3.17 & 17.30 \\
\hline
\end{tabular}

transform models, SLD and EUL, exhibit a narrower precipitation profile, whereas the distributions in the two grid-pointbased dynamical cores, FV and SE, occupy a slightly wider latitudinal range.

These tropical precipitation rates in MITC allow for further process studies. From theory, it is expected that the large-scale precipitation rates are closely connected to the horizontal convergence of the lower-level winds, which in turn determines the strength of the updrafts at lower levels in the ITCZ region. These dynamical connections are displayed in Figs. 12 and 13. In particular, Fig. 12 demonstrates that the equatorial precipitation rate is strongest in SLD, followed by EUL, FV, and SE. As a consequence, the resulting equatorial updrafts in Fig. 13 are therefore strongest in SLD, followed by EUL and the rather similar updraft speeds in FV and SE. Because precipitation is favored in regions with abundant moisture (present in all dynamical cores) and strong updrafts this explains the differing strengths of the equatorial precipitation rates. As an aside, once precipitation is enhanced in SLD, the latent heating from precipitation is increased and further supports strong updrafts and lower-level convergence. This is a positive feedback loop that was also discussed for self-enforcing grid-point storms by Williamson (2013).

Figures 12 and 13 also confirm the earlier MITC finding that the two spectral transform models, SLD and EUL, exhibit a narrower ITCZ profile than the grid-point-based dynamical cores, FV and SE. This is displayed by the narrower regions of equatorial precipitation, or equivalently the steeper slopes in the precipitation rate curves, in SLD and EUL. Additionally, SLD and EUL exhibit stronger low-level downdrafts between \pm 12 to $\pm 5^{\circ}$, which reside in the descending branch of the Hadley circulation (Fig. 13c and d). In summary, Figs. 12 and 13 demonstrate that the Hadley circulation in MITC simulations is more vigorous in SLD and EUL with narrower and more intense updraft zones at the Equator.

This raises the question whether the numerical designs of the dynamical cores contribute to the differing strengths of the Hadley circulations. The spectral transform method represents a global numerical discretization where the flow at 


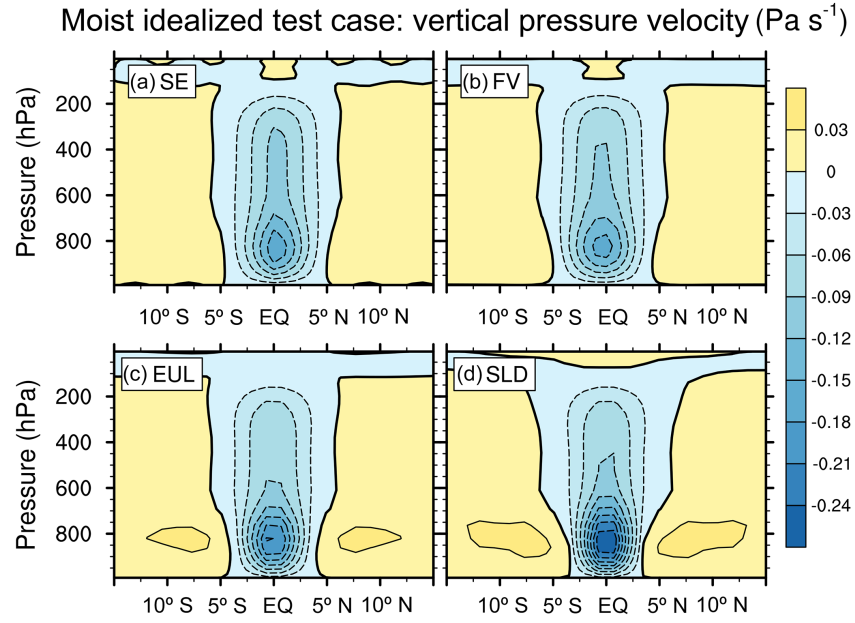

Figure 13. Latitude-pressure profiles of time-mean, zonal-mean vertical pressure velocity in MITC for (a) SE, (b) FV, (c) EUL, and (d) SLD.

each point depends on all other grid points. The grid-pointbased models are built upon local numerical discretizations that only rely on nearest-neighbor information. However, the question whether these numerical design differences systematically impact the Hadley circulation cannot be determined from these four model examples and will require additional model intercomparisons. It is interesting though that the MITC precipitation and Hadley circulation characteristics are not fully replicated by their CAM5 aquaplanet counterparts (not shown in detail, but see Table 3). Once more complex moist interactions are included in the APS experiments the maximum precipitation and updraft strength are exhibited by $\mathrm{FV}$ with a mean equatorial peak precipitation rate (PRECT) of around $20 \mathrm{~mm} \mathrm{day}^{-1}$ as listed in Table 3.

In addition, the APS PRECT distributions in EUL, SLD, and SE (shown earlier in Fig. 4b) almost overlay each other with slightly different mean equatorial peaks between 17.3 and 19.2 mm day $^{-1}$ (see Table 3). This demonstrates that the peak APS total precipitation rates at the Equator are quite similar to their MITC variants, but that the interaction with the CAM5 physical parameterizations changes the relative order of the four dynamical cores. In addition, the time-mean global-mean precipitation rates are bigger in APS (about $3.2 \mathrm{~mm} \mathrm{day}^{-1}$ ) than in MITC (about $2.2 \mathrm{~mm} \mathrm{day}^{-1}$ ) due to the more elaborate hydrological cycle in aquaplanet simulations. Such differences between the MITC and APS simulations need to be expected and are mainly caused by the presence of the shallow and deep convection parameterizations and the more effective boundary-layer mixing in APS. Their presence also strongly modulates the widths and strengths of the Hadley circulation as shown earlier for CAM5-SE (Figs. 2a-c and 4).

As an aside, the overall similarities between the four CAM5 dynamical cores in the MITC and APS configurations are quite remarkable. This is in sharp contrast to the APE simulations documented by Williamson et al. (2012) and Blackburn et al. (2013), who intercompared 16 aquaplanet configurations with different dynamical cores and different physical parameterizations. For example, their time-mean, zonal-mean, and hemispherically averaged total precipitation rates exhibit equatorial peaks between 10 and $34 \mathrm{~mm} \mathrm{day}^{-1}$ (Fig. 4 in Blackburn et al., 2013). This strongly suggests that the likely more subtle differences between the dynamical cores (as seen in MITC and APS) can easily be overshadowed by a more dominant impact of the varying physical parameterizations in the APE simulations. This again demonstrates the importance of well-designed idealized test cases that distinguish between causes and effects. Note that some APE models in Blackburn et al. (2013) develop a double ITCZ that is not present in the CAM5 MITC and APS simulations shown here. However, when changing some CAM5 physical parameterizations double ITCZs can also be generated, which furthermore depend on the diffusion properties of the dynamical cores. This is not further discussed here but showcases how idealized model configurations such as MITC and APS can reveal these model intricacies and their dynamical core impact. On a final note, the MITC and APE experiments (with single ITCZs) have one important aspect in common. The higher the equatorial precipitation rate the narrower the ITCZ region, which can be seen in our Fig. 12 and Fig. 4 in Blackburn et al. (2013).

In addition to the mean precipitation statistics, the frequency distribution of the precipitation rates for all four MITC dynamical cores is shown in Fig. 14. In particular, the figure displays the fraction of the precipitation rate between $10^{\circ} \mathrm{N}$ and $10^{\circ} \mathrm{S}$ that falls into each precipitation bin. As seen in Sect. 4.3, the assessment is based on 6 months of 6-hourly instantaneous precipitation data that have been conservatively regridded to a $2^{\circ} \times 2^{\circ}$ latitude-longitude grid before the analysis. The precipitation rates in Fig. 14a range from 0 to $120 \mathrm{~mm} \mathrm{day}^{-1}$ with $1 \mathrm{~mm} \mathrm{day}^{-1}$ bins, and from 0 to $600 \mathrm{~mm} \mathrm{day}^{-1}$ with $10 \mathrm{~mm} \mathrm{day}^{-1}$ bins in Fig. $14 \mathrm{~b}$.

Figure 14a indicates that all four dynamical cores have similar distributions for low precipitation rates with curves that mostly overlay each other. However, above $200 \mathrm{~mm} \mathrm{day}^{-1}$ the range of distributions widens as seen in Fig. 14b. In particular, SE and EUL show the most frequent extreme precipitation events that range up to $550-600 \mathrm{~mm} \mathrm{day}^{-1}$. This is followed by the FV dynamical core with peak events around $450 \mathrm{mmday}^{-1}$, whereas the extreme precipitation rates in SLD only reach values around $350 \mathrm{~mm} \mathrm{day}^{-1}$. Extreme precipitation events are closely connected to grid-scale-size storms, as investigated by Williamson (2013), that are closely linked to extreme vertical pressure velocities. The latter have been shown in Fig. 9c for SE (se_ftype =1) and in Fig. 10 for FV, EUL, and SLD in MITC mode. From these, it is obvious that both SE and EUL show the most intense $850 \mathrm{hPa}$ vertical pressure velocities in the tropical region between $\pm 10^{\circ}$, whereas the 

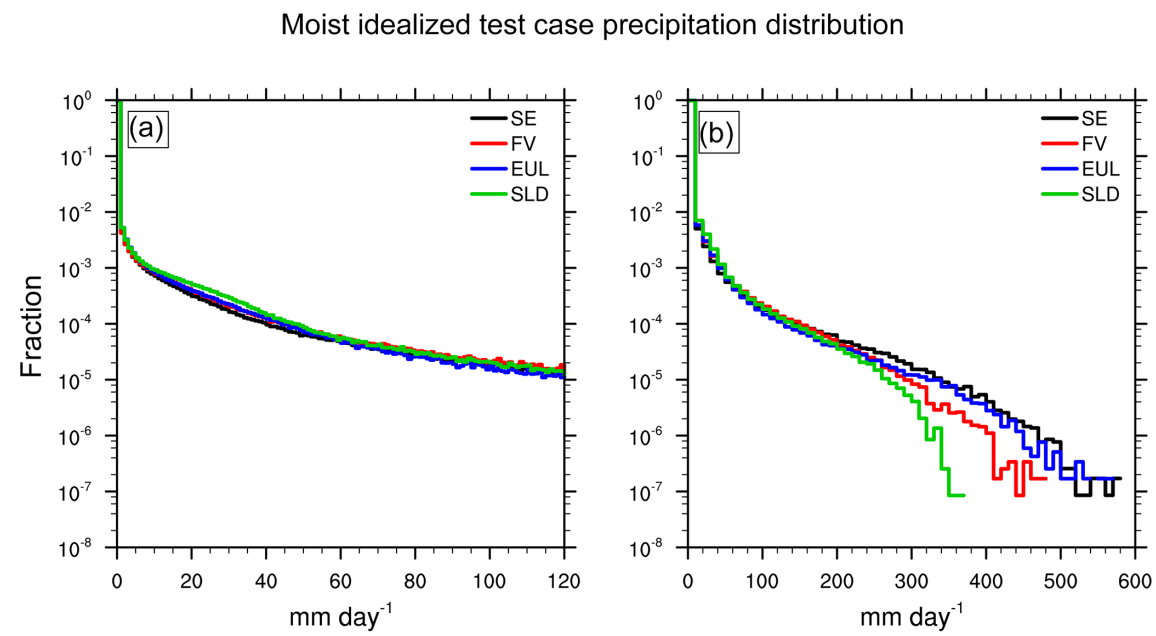

Figure 14. Frequency distributions of the MITC precipitation rates averaged between $\pm 10^{\circ}$ for SE (black), FV (red), EUL (blue), and SLD (green). (a) Precipitation rates range from 0 to $120 \mathrm{~mm} \mathrm{day}^{-1}$ with $1 \mathrm{~mm} \mathrm{day}^{-1}$ bins, (b) precipitation rates range from 0 to $600 \mathrm{~mm} \mathrm{day}{ }^{-1}$ with $10 \mathrm{~mm}$ day $^{-1}$ bins.

tropical vertical pressure velocities are somewhat reduced in FV and SLD. Furthermore, the occurrences of the grid-pointscale storms in FV and SLD are visibly reduced in these instantaneous $\omega$ snapshots.

As a point of comparison, Fig. 15 displays the corresponding precipitation frequency distributions of all four APS configurations. The two MITC model outliers are replicated by the APS results. In particular, the most extreme tropical precipitation events are present in CAM5-SE whereas the least active dynamical core is SLD. The peak magnitudes of the heavy rainfall events are reduced to 420 and $240 \mathrm{~mm}$ day $^{-1}$ in SE and SLD, respectively, which is a result of the deep convection parameterization in the APS simulations. The SE distribution is closely tracked by the FV dynamical core with peak precipitation events around $380 \mathrm{~mm} \mathrm{day}^{-1}$. EUL's peak precipitation rates in the APS configuration reaches about $320 \mathrm{~mm}$ day $^{-1}$. While the APS and MITC frequency distributions are not a perfect match, as expected, they do reveal the general characteristics of the physics-dynamics coupling strategy and the likelihood of grid-scale-size storms.

\subsubsection{Equatorial waves}

The final analyses focus on the tropical wave activity in the four MITC simulations, which can also be compared to the CAM5-SE discussions in Sect. 4.4. The convectively coupled equatorial waves are again analyzed via the Wheeler and Kiladis (1999) wavenumber-frequency spectra. As before, the analysis is based on 6 months of 6-hourly instantaneous $100 \mathrm{hPa}$ temperature data between $15^{\circ} \mathrm{S}$ and $15^{\circ} \mathrm{N}$, using consecutive 96 day windows with 60 days of overlap.

Figure 16 shows the symmetric wavenumber-frequency spectra of all four dynamical cores in the MITC configuration. The solid lines are dispersion curves that indicate lines of constant equivalent depth with $h=12,25$, and $50 \mathrm{~m}$. Furthermore, the thick dashed line denotes the $h=200 \mathrm{~m}$ dispersion curve. All dynamical cores have prominent, eastward propagating Kelvin waves that have, in general, similar characteristics. SE and FV (Fig. 16a and b) both feature Kelvin waves with equivalent depths of slightly larger than $50 \mathrm{~m}$ at a wide range of frequencies. EUL and SLD (Fig. 16c and d) both feature Kelvin waves with an equivalent depth below $50 \mathrm{~m}$ and predominantly shorter frequencies (longer periods) than SE and FV. It seems as if the overall Kelvin wave activity in SLD is least abundant. This could be related to the reduced occurrences of intense precipitation rates as displayed in Fig. 14b.

Convectively uncoupled Kelvin waves with equivalent depths of around $200 \mathrm{~m}$ are also present in all four dynamical cores as further described in Sect. 4.4. These high-speed Kelvin waves seem to be most abundant in the EUL simulation and occupy a wider high-frequency range. This aspect might be connected to the EUL's gravity wave noise, discussed earlier in Sect. 5.2.1, but this link needs further investigations.

\section{Suggested further extensions of the MITC approach}

The MITC can be considered a test case of intermediate complexity that is highly versatile in its present form as demonstrated in Sects. 4 and 5. However, many other model configurations or application areas are feasible. For example, the complexity of the MITC physical parameterizations and included processes can be increased, including the inclusion of a deep convection scheme, the use of a Kessler-type warmrain scheme instead of large-scale condensation, the inclusion of idealized topography as "water mountains", the in- 
Aquaplanet precipitation distribution
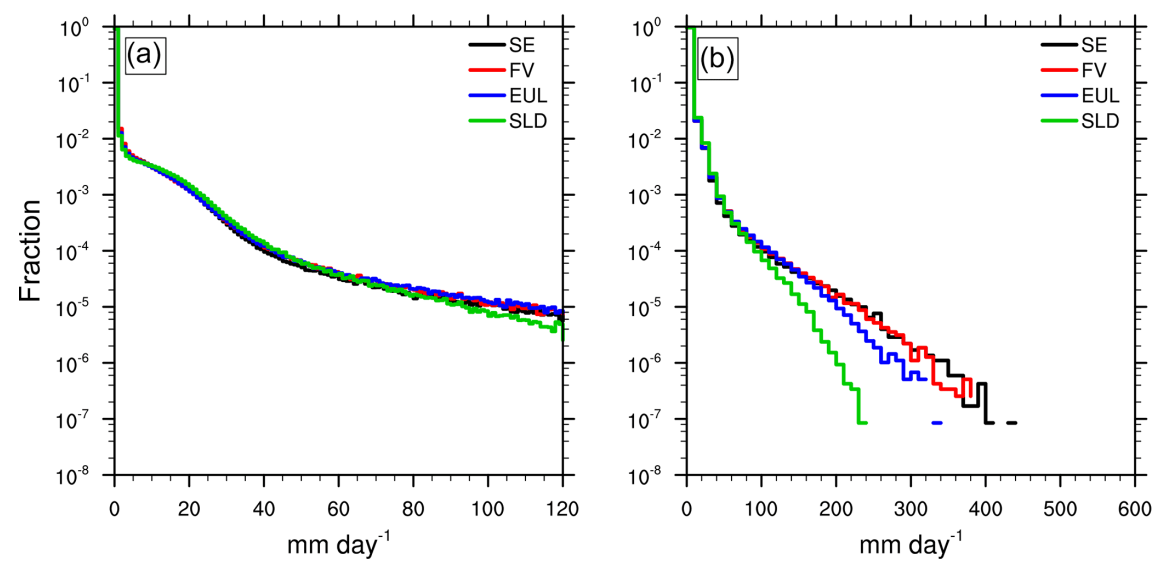

Figure 15. Same as Fig. 14 but for the CAM5 APS configurations of SE (black), FV (red), EUL (blue), and SLD (green).

clusion of a land-sea mask with dry land points, or the replacement of the prescribed SSTs with a slab ocean model with a constant mixed-layer depth. Furthermore, many other application areas are feasible, such as a systematic study of tropical waves and the stratospheric quasi-biennial oscillation under varying moisture conditions, grid imprinting aspects of non-latitude-longitude grids or computational grids with variable resolution, more-in-depth analyses of the physics-dynamics coupling interface and numerical noise, as well as community-wide moist dynamical core model intercomparisons. Here we briefly characterize these possible enhancements that all warrant further research.

\subsection{Addition of a deep convection scheme}

The inclusion of a convection scheme to a simplified physics framework was for example demonstrated by Frierson (2007b), who formulated a simplified version of a BettsMiller-type convective adjustment parameterization. We already experimented with this simplified Betts-Miller convection parameterization in the MITC, which we called before the large-scale condensation. While the inclusion of this parameterization moved the MITC simulations closer to the APS results with broader ITCZs and enhanced upper level heating in the tropical atmosphere (not shown), we found the simulations to be rather sensitive to the subjective choice of the Betts-Miller relative humidity threshold and relaxation timescale. These two convection parameters needed to vary considerably in the CAM5 dynamical cores for comparable results. This is consistent with the findings in Frierson (2007a), who demonstrated that the variation of the two parameters greatly impacted the Kelvin wave activity in his simplified-physics framework. It highlights the complex nonlinear interactions between the dynamical core and the moist processes.

\subsection{Use of a Kessler-type warm-rain scheme}

The large-scale condensation in MITC can also be replaced with a more complex warm-rain Kessler-type physical parameterization that was, e.g., detailed in Klemp and Wilhelmson (1978). The Kessler-type parameterization is an example of a cloud microphysics scheme. It adds cloud water and rain water to the list of prognostic variables, and incorporates new processes such as the autoconversion and accretion of cloud water to rain. A Kessler-type example Fortran routine has recently been provided by Klemp et al. (2015). Note though that this implementation implicitly assumes very short physics time steps (on the order of seconds) in order to obey a numerical stability constraint in the vertical direction. More specifically, the rain drop fall speed on the order of $5-6 \mathrm{~m} \mathrm{~s}^{-1}$ is only allowed to transport rain to the underlying grid box, which might be located in close proximity depending on the vertical grid spacing (typically below $100 \mathrm{~m}$ at low levels). A more practicable approach for physics time steps of order $1800 \mathrm{~s}$ is therefore the inclusion of a rainfall sub-cycling scheme, which is present in the Kessler-type Fortran routine in the Weather Research and Forecasting Model (WRF) (Skamarock et al., 2008). We recently included the Kessler-type warm rain scheme with subcycling in MITC-CAM and found that the general circulation is almost identical to the study presented here.

\subsection{Inclusion of water mountains}

The current MITC configuration assumes a water-covered planet with zero surface elevation that forces the general circulation to be symmetric in the Northern and Southern hemispheres. However, idealized or even realistic water-covered mountains can be introduced and were denoted as "water mountains" by Schneider et al. (2015). They impact the general circulation, for example via the generation of topo- 

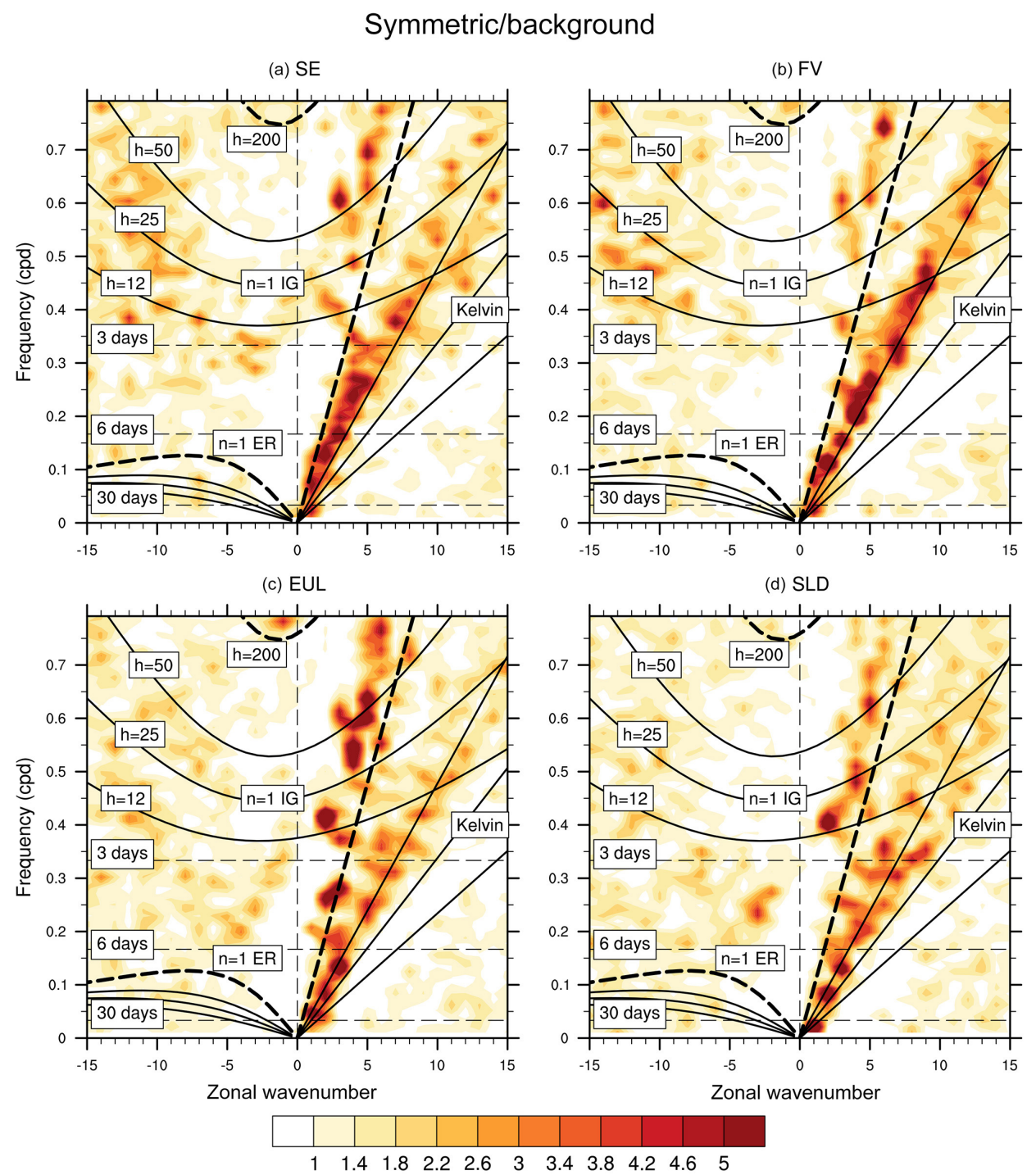

Figure 16. Wavenumber-frequency diagrams for the MITC simulations showing spectral power for the symmetric component of the $100 \mathrm{hPa}$ equatorial temperature averaged between $\pm 15^{\circ}$ for (a) SE, (b) FV, (c) EUL, and (d) SLD. The solid lines show the theoretical shallow-water dispersion curves for the equivalent depths $h=12,25$, and $50 \mathrm{~m}$ without a mean background velocity. The thick dashed line is the $h=200 \mathrm{~m}$ dispersion curve.

graphic rain, and lead to hemispherically asymmetric circulations. However, such a variation needs a careful review of the lower boundary condition. Schneider et al. (2015) used a slab ocean configuration that self-adjusts its surface temperature based on the surface energy balance. In MITC, the SSTs are prescribed and will need to be height adjusted to reasonably mimic the vertical temperature variations above topography.

\subsection{Impact of land-sea masks}

The current MITC configuration has no information about land-sea contrasts. All grid points are water-covered and allow for the evaporation of water via the latent heat flux at the surface. A straightforward modification of the MITC approach is the introduction of a land-sea mask with dry or reduced-moisture land points without any surface elevation. This can easily be accomplished by suppressing or reducing the surface latent heat flux over land areas. It will lead to hemispherically asymmetric circulations and should have an impact on the wave activity.

\subsection{Slab ocean configurations}

As mentioned in the introduction, alternative simplified physics packages such as the ones by Frierson et al. (2006) and O'Gorman and Schneider (2008) include mixed-layer oceans with constant mixed-layer depths that close the en- 
ergy budget at the lower boundary. This is imperative for idealized climate change studies in order to obtain energetically consistent changes in precipitation. A consequence of the slab ocean configuration is the use of a more complicated radiation parameterization that gives access to the net shortwave and incoming and outgoing longwave radiation at the lower boundary. The MITC approach can be converted to a slab ocean configuration without prescribed SSTs. However, careful adjustments of the radiation need to be considered and will likely require the replacement of the Newtonian temperature relaxation for this configuration.

\subsection{Varying moisture conditions for tropical wave and stratospheric QBO studies}

Recently, Yao and Jablonowski $(2013,2015)$ detected that dry Held-Suarez experiments support the generation of tropical stratospheric oscillations that closely resemble the stratospheric QBO. Because QBOs are wave-driven phenomena, the wave triggering and transport characteristics of the dynamical cores are of critical importance and determine the magnitude and period of the QBO-like oscillations. The MITC, as the moist variant of the HS configuration, therefore gives further access to an improved understanding of the wave-mean flow interaction. Because resolved-scale convection and rain act as effective tropical wave generators, the QBO-like oscillations in the four CAM5 dynamical cores are greatly impacted. In particular, the enhanced wave forcing due to moisture processes lead to shorter QBO periods and enhanced magnitudes in some sample MITC studies (not shown here). The moisture content of the MITC atmospheres can also be easily varied, e.g., via the adjustment of the bulk transfer coefficients for latent heat $C_{E}$ or the variation of the saturation vapor pressure $e_{0}^{*}$ coefficient as done in Frierson et al. (2006). This allows for systematic studies of the tropical wave activity, the wave-mean flow interactions, and their dependence on the hydrological cycle.

\subsection{Grid imprinting}

The MITC can also be used in its present form to further investigate grid imprinting issues. These might arise from the use of non-latitude-longitude computational grids in the dynamical cores, such as the CAM5-SE cubed-sphere configuration. Cubed-sphere grids have built-in wavenumber four irregularities in both hemispheres; therefore, the question arises whether these mesh characteristics can be detected in the general circulation. An example of such grid imprinting assessments with the dry Held-Suarez test was provided by Harris and Lin (2013), who found cubed-sphere wavenumber four anomalies in the time-mean vertical pressure velocities at low and mid-levels. They also found grid imprinting signatures in the variable-resolution configuration of their model. Such grid imprinting issues are likely to become more severe once moist interactions are included, and the MITC pro- vides an idealized test bed for such investigations. Due to the simplistic nature of the large-scale condensation, the MITC is expected to exhibit some grid-size sensitivities. Therefore, this allows for more enhanced assessments, such as the grid-scale sensitivities of newly added convection schemes that promise to be more scale-aware (e.g., Grell and Freitas, 2014).

\subsection{Physics-dynamics coupling interfaces}

As shown in Sect. 5, the MITC serves as an idealized test bed for physics-dynamics coupling strategies because it replicates the coupling intricacies of complex-physics simulations. Additional application examples are suggested, such as the sensitivities of the coupling to the physics time step that was recently investigated by Wan et al. (2015). They conducted numerical convergence studies with respect to shrinking physics time steps in CAM5-SE and used the MITC/RJ12 large-scale condensation as a baseline example for the convergence behavior. Furthermore, parallel- vs. sequential-split coupling strategies can be investigated in the MITC simplified framework before complex physical parameterizations are assessed. In addition, MITC allows for an in-depth analysis of other dynamics-physics interactions such as the impact of varying explicitly added or implicit numerical diffusion on extreme precipitation statistics. As shown in Jablonowski and Williamson (2011) (their Sect. 13.4.1.2 and their Fig. 13.8) an increase in the horizontal divergence damping can have a profound impact on the frequency distribution of the tropical precipitation rate. In particular, an increase in the diffusion led to a sharp decrease in the likelihood of heavy precipitation events in CAM3.5-FV aquaplanet configurations at low resolutions.

\subsection{Community-wide moist dynamical core intercomparisons}

The snapshots of the CAM5 dynamical core intercomparison presented here provide a first glimpse of the potential model spread and model uncertainty in the presence of nonlinear moisture feedbacks. Other dynamical core configurations need to be tested, such as newly emerging nonhydrostatic dynamical cores, dynamical cores with deepatmosphere configurations, and models with other numerical discretizations and computational grids. This will provide further insights into the model spread and might even serve as a debugging and learning tool for models in their early development stages. Therefore, we encourage the incorporation of the easy-to-use MITC approach into the routine test beds of the dynamical cores and the documentation of the results in the literature. This will establish a broad base for model intercomparisons and help establish standards. 


\section{Conclusions}

A moist idealized test case for atmospheric model dynamical cores has been presented. This test case was inspired by the dry Held-Suarez test and the simplified physics package of RJ12. This new variant of the HS test includes moisture and thereby sheds light on the nonlinear dynamics-physics moisture feedbacks without the complexity of full-physics parameterization packages. In particular, it adds simplified moist processes to the modified HS temperature relaxation and low-level HS Rayleigh friction to model large-scale condensation, boundary-layer mixing, and the exchange of latent and sensible heat between the atmospheric surface and an ocean-covered planet with prescribed sea surface temperatures. Using the CAM5-SE dynamical core we demonstrate that the inclusion of the moist idealized physics package leads to climatic states that closely resemble aquaplanet simulations with complex physical parameterizations. Comparisons to both APS and APS without deep convection are provided to further shed light on the role of the deep convection parameterization and its moisture transport.

We establish that the MITC approach generates reasonable atmospheric circulations that serve as a useful test bed and application tool for a broad range of scientific investigations. Two example application areas were presented. First, we showed that the test case reveals the characteristics of the physics-dynamics coupling technique and reproduces coupling issues seen in full-physics simulations. In particular, we demonstrated in CAM5-SE that sudden adjustments of the prognostic fields due to moist physics tendencies can trigger undesirable large-scale gravity waves, which can be remedied by a more gradual application of the physical forcing. Second, the moist idealized test case was used for a dynamical core intercomparison that is based on the four CAM5 dynamical cores SE, FV, EUL, and SLD. These represent a wide selection of numerical approaches and show their impact on the general circulation.

In general, we found that the moist dynamical cores generate similar climatic states, which is especially true in the midlatitudes and polar regions. The differences are most pronounced in the tropical regions that experience the biggest impact from the moist physical parameterizations. In particular, the MITC simulations revealed differing character- istics of the ITCZs and Hadley circulations, and furthermore provided insight into the likelihood of extreme precipitation events. The latter are impacted by the occurrences of gridpoint-size storms, which gives further information about the intricacies of the physics-dynamics coupling. Selected comparisons to aquaplanet simulations demonstrate that some MITC characteristics almost perfectly replicate their APS counterparts, such as the shapes of the upper-tropospheric kinetic energy spectra. The steepness of the kinetic energy slopes is closely connected to the diffusion characteristics of the dynamical cores; therefore, it suggests that the dynamical cores are the dominating factors for these KE assessments. Precipitation-related processes do not typically exhibit a perfect match between MITC and APS. However, this is neither expected nor required to serve as a useful test bed. It was shown that the overall characteristics like the equatorial total precipitation rates in MITC and APS were rather similar, and that the least active and most active dynamical core with respect to the extreme tropical precipitation are identical in MITC and APS simulations. All dynamical cores trigger convectively coupled equatorial waves despite the simplicity of the MITC package. This allows for a detailed assessment of the tropical wave activity.

MITC helps fill the gap in the current hierarchy of GCM test cases, which lacks easy-to-use, fully documented, and computationally inexpensive configurations like the MITC. The MITC simulations distinguish between causes and effects, and our goal is to establish standards for moist dynamical core assessments. The dynamical cores used here represent a variety of commonly used numerical methods and grid designs for atmospheric general circulation models. It is shown that the moist idealized test case is robust enough to generate similar climates in all four dynamical cores, yet sensitive enough to reveal their differences. Further work should explore these differences in greater detail, including statistical analyses of the differences between the four CAM5 dynamical cores and additional comparisons to dynamical cores from other GCMs. In addition, many extensions of the MITC approach and a wide array of other application areas are feasible, which we suggest exploring in the future. 


\section{Appendix A}

The MITC simulations presented in this paper are all initialized and spun up with an identical initial state, which we document for completeness. It is based on the shallowatmosphere version of the baroclinic wave test for dynamical cores developed by Ullrich et al. (2014). This set of initial conditions provides a steady-state atmosphere with constant zero surface geopotential, or no topography. The moist surface pressure is set to $p_{\mathrm{s}}=p_{0}=1000 \mathrm{hPa}$ everywhere. This is slightly lower than the recommended moist $p_{\mathrm{s}}$ value for the APE simulations (1013.25 hPa). The initial state also includes a perturbation in the zonal-wind field, which generates synoptic-scale waves in the Northern Hemisphere midlatitudes. This baroclinic wave test is designed for dry dynamical cores, therefore moisture has been added. The specific humidity profile is calculated by

$$
\begin{aligned}
& q(\phi, \sigma)= \\
& \begin{cases}q_{0} \exp \left[-\left(\frac{\phi}{\phi_{\mathrm{hw}}}\right)^{4}\right] \exp \left[-\left((\sigma-1)\left(\frac{p_{0}}{p_{\mathrm{hw}}}\right)\right)^{2}\right] & p \geq 100 \mathrm{hPa}, \\
0 & p<100 \mathrm{hPa}\end{cases}
\end{aligned}
$$

where $q_{0}=18 \mathrm{~g} \mathrm{~kg}^{-1}$ is the maximum specific humidity, $\phi_{\mathrm{hw}}=2 \pi / 9$ radians $\left(40^{\circ}\right)$ is the horizontal half-width of the specific humidity profile with latitude, and $p_{\mathrm{hw}}=300 \mathrm{hPa}$ is the vertical half-width of the specific humidity profile with pressure. In this moist environment the initial temperature profile (Eq. 20 in Ullrich et al., 2014) now represents the virtual temperature, $T_{\mathrm{v}}$, and the temperature profile is calculated by

$T=\frac{T_{\mathrm{v}}}{1+0.608 q}$.

The resulting initial conditions (see complete equation set in Ullrich et al., 2014) form a stable atmosphere that is suitable for initializing the moist idealized test. Other initial conditions may also be used. We advise against using a dry initial state because large physics tendencies could cause the model to become unstable at the beginning of the model run. An appropriate spin up time, such as 6 months in our simulations, should allow the atmosphere to stabilize before the results are used for analysis. 


\section{The Supplement related to this article is available online at doi:10.5194/gmd-9-1263-2016-supplement.}

Acknowledgements. We thank the reviewers for their valuable suggestions that improved the manuscript. This work was supported by the US Department of Energy (DoE), Office of Science, award nos. DE-SC0006684 and DE-SC0003990. We acknowledge the high-performance computing support from Yellowstone (ark:/85065/d7wd3xhc) provided by NCAR's Computational and Information Systems Laboratory, sponsored by the National Science Foundation.

Edited by: V. Grewe

\section{References}

Baldauf, M., Reinert, D., and Zängl, G.: An analytical solution for linear gravity and sound waves on the sphere as a test for compressible, non-hydrostatic numerical models, Q. J. Roy. Meteor. Soc., 140, 1974-1985, doi:10.1002/qj.2277, 2014.

Blackburn, M., Williamson, D. L., Nakajima, K., Ohfuchi, W., Takahashi, Y. O., Hayashi, Y. Y., Nakamura, H., Ishiwatari, M., McGregor, J. L., Borth, H., Wirth, V., Frank, H., Bechtold, P., Wedi, N. P., Tomita, H., Satoh, M., Zhao, M., Held, I. M., Suarez, M. J., Lee, M. I., Watanabe, M., Kimoto, M., Liu, Y., Wang, Z., Molod, A., Rajendran, K., Kitoh, A., and Stratton, R. A.: The Aqua-Planet Experiment (APE): CONTROL SST simulation, J. Meteorol. Soc. Jpn., 91A, 17-56, doi:10.2151/jmsj.2013-A02, 2013.

Chen, C.-T. and Knutson, T.: On the verification and comparison of extreme rainfall indices from climate models, J. Climate, 21, 1605-1621, doi:10.1175/2007JCLI1494.1, 2008.

Chen, M., Rood, R. B., and Takacs, L. L.: Impact of a semiLagrangian and an Eulerian dynamical core on climate simulations, J. Climate, 10, 2374-2389, 1997.

Claussen, M., Mysak, L., Weaver, A., Crucifix, M., Fichefet, T., Loutre, M.-F., Weber, S., Alcamo, J., Alexeev, V., Berger, A., Calov, R., Ganopolski, A., Goose, H., Lohmann, G., Lunkeit, F., Mokhov, I. I., Petoukhov, V., Stone, P., and Wang, Z.: Earth system models of intermediate complexity: closing the gap in the spectrum of climate system models, Clim. Dynam., 18, 579-586, 2002.

Dennis, J. M., Edwards, J., Evans, K. J., Guba, O., Lauritzen, P. H., Mirin, A. A., St-Cyr, A., Taylor, M. A., and Worley, P. H.: CAMSE: a scalable spectral element dynamical core for the Community Atmosphere Model, Int. J. High Perform. C., 26, 74-89, doi:10.1177/1094342011428142, 2012.

Evans, K. J., Lauritzen, P., Mishra, S., Neale, R., Taylor, M., and Tribbia, J.: AMIP simulation with the CAM4 spectral element dynamical core, J. Climate, 26, 689-709, doi:10.1175/JCLI-D11-00448.1, 2013.

Fournier, A., Taylor, M. A., and Tribbia, J. J.: The spectral element atmosphere model (SEAM): high-resolution parallel computation and localized resolution of regional dynamics, Mon. Weather Rev., 132, 726-748, doi:10.1175/15200493(2004)132<0726:TSEAMS>2.0.CO;2, 2004.
Frierson, D. M.: Convectively coupled Kelvin waves in an idealized moist general circulation model, J. Atmos. Sci., 64, 2076-2090, 2007a.

Frierson, D. M.: The dynamics of idealized convection schemes and their effect on the zonally averaged tropical circulation, J. Atmos. Sci., 64, 1959-1976, doi:10.1175/JAS3935.1, 2007b.

Frierson, D. M., Held, I. M., and Zurita-Gotor, P.: A gray-radiation aquaplanet moist GCM. Part I: Static stability and eddy scale, J. Atmos. Sci., 63, 2548-2566, doi:10.1175/JAS3753.1, 2006.

Galewsky, J., Sobel, A., and Held, I.: Diagnosis of subtropical humidity dynamics using tracers of last saturation, J. Atmos. Sci., 62, 3353-3367, doi:10.1175/JAS3533.1, 2005.

Gates, W. L., Boyle, J. S., Covey, C. C., Dease, C. G., Doutriaux, C. M., Drach, R. S., Fiorino, M., Gleckler, P. J., Hnilo, J. J., Marlais, S. M., Phillips, T. J., Potter, G. L., Santer, B. D., Sperber, K. R., Taylor, K. E., and Williams, D. N.: An overview of the results of the Atmospheric Model Intercomparison Project (AMIP I), B. Am. Meteorol. Soc., 80, 29-55, 1999.

Grabowski, W. W. and Smolarkiewicz, P. K.: A multiscale anelastic model for meteorological research, Mon. Weather Rev., 130, 939-956, doi:10.1175/15200493(2002)130<0939:AMAMFM>2.0.CO;2, 2002.

Grell, G. A. and Freitas, S. R.: A scale and aerosol aware stochastic convective parameterization for weather and air quality modeling, Atmos. Chem. Phys., 14, 5233-5250, doi:10.5194/acp-145233-2014, 2014.

Gross, M., Malardel, S., Jablonowski, C., and Wood, N.: Bridging the (knowledge) gap between physics and dynamics, B. Am. Meteorol. Soc., doi:10.1175/BAMS-D-15-00103.1, in press, 2016.

Harris, L. M. and Lin, S.-J.: A two-way nested global-regional dynamical core on the cubed-sphere grid, Mon. Weather Rev., 141, 283-306, 2013.

Held, I. M.: The gap between simulation and understanding in climate modeling, B. Am. Meteorol. Soc., 86, 1609-1614, doi:10.1175/BAMS-86-11-1609, 2005.

Held, I. M. and Suarez, M. J.: A proposal for the intercomparison of the dynamical cores of atmospheric general circulation models, B. Am. Meteorol. Soc., 75, 1825-1830, doi:10.1175/15200477(1994)075<1825:APFTIO>2.0.CO;2, 1994.

Jablonowski, C.: Test der Dynamik zweier globaler Wettervorhersagemodelle des Deutschen Wetterdienstes: Der Held-Suarez Test, Master's thesis, University of Bonn, Germany, Department of Meteorology, 140 pp., 1998.

Jablonowski, C. and Williamson, D. L.: A baroclinic instability test case for atmospheric model dynamical cores, Q. J. Roy. Meteor. Soc., 132, 2943-2975, doi:10.1256/qj.06.12, 2006.

Jablonowski, C. and Williamson, D. L.: The pros and cons of diffusion, filters and fixers in atmospheric general circulation models, in: Numerical Techniques for Global Atmospheric Models, edited by: Lauritzen, P. H., Jablonowski, C., Taylor, M. A., and Nair, R. D., Vol. 80 of Lecture Notes in Computational Science and Engineering, Springer, 381-493, 2011.

Kent, J., Ullrich, P. A., and Jablonowski, C.: Dynamical core model intercomparison project: tracer transport test cases, Q. J. Roy. Meteor. Soc., 140, 1279-1293, 2014.

Klemp, J. B. and Wilhelmson, R. B.: The simulation of threedimensional convective storm dynamics, J. Atmos. Sci., 35, 1070-1096, 1978. 
Klemp, J. B., Skamarock, W. C., and Park, S.-H.: Idealized global nonhydrostatic atmospheric test cases on a reduced radius sphere, J. Adv. Model. Earth Syst., 7, 155-1177 doi:10.1002/2015MS000435, 2015.

Kurowski, M. J., Grabowski, W. W., and Smolarkiewicz, P. K.: Anelastic and compressible simulation of moist dynamics at planetary scales, J. Atmos. Sci., 72, 3975-3995, 2015.

Lauritzen, P. H., Jablonowski, C., Taylor, M. A., and Nair, R. D.: Rotated versions of the Jablonowski steady-state and baroclinic wave test cases: a dynamical core intercomparison, J. Adv. Model. Earth Syst., 2, 15, doi:10.3894/JAMES.2010.2.15, 2010.

Lin, S.-J.: A "vertically Lagrangian" finite-volume dynamical core for global models, Mon. Weather Rev., 132, 2293-2307, doi:10.1175/1520-0493(2004)132<2293:AVLFDC>2.0.CO;2, 2004

Lin, S.-J. and Rood, R. B.: Multidimensional fluxform semi-Lagrangian transport schemes, Mon. Weather Rev., 124, 2046-2070, doi:10.1175/15200493(1996)124<2046:MFFSLT>2.0.CO;2, 1996.

Lin, S.-J. and Rood, R. B.: An explicit flux-form semi-Lagrangian shallow-water model on the sphere, Q. J. Roy. Meteor. Soc., 123, 2477-2498, doi:10.1002/qj.49712354416, 1997.

Malardel, S.: Physics/Dynamics Coupling, ECMWF Workshop on Non-hydrostatic Modelling, ECMWF, 8-10 November 2010, Reading, United Kingdom, 67-77, 2011.

McCalpin, J. D.: A quantitative analysis of the dissipation inherent in semi-Lagrangian advection, Mon. Weather Rev., 116, 2330 2336, 1988 .

Medeiros, B., Stevens, B., Held, I. M., Zhao, M., Williamson, D. L., Olson, J. G., and Bretherton, C. S.: Aquaplanets, climate sensitivity, and low clouds, J. Climate, 21, 4974-4991, doi:10.1175/2008JCLI1995.1, 2008.

Mishra, S. K., Taylor, M. A., Nair, R. D., Tufo, H. M., and Tribbia, J. J.: Performance of the HOMME dynamical core in the aquaplanet configuration of NCAR CAM4: equatorial waves, Ann. Geophys., 29, 221-227, doi:10.5194/angeo-29-221-2011, 2011a.

Mishra, S. K., Taylor, M. A., Nair, R. D., Lauritzen, P. H., Tufo, H. M., and Tribbia, J. J.: Evaluation of the HOMME dynamical core in the aquaplanet configuration of NCAR CAM4: rainfall, J. Climate, 24, 4037-4055, doi:10.1175/2011JCLI3860.1, 2011b.

Mitchell, H. L., Houtekamer, P., and Pellerin, G.: Ensemble size, balance, and model-error representation in an ensemble Kalman filter, Mon. Weather Rev., 130, 2791-2808, doi:10.1175/15200493(2002)130<2791:ESBAME>2.0.CO;2, 2002.

Nastrom, G. D. and Gage, K. S.: A climatology of atmospheric wavenumber spectra of wind and temperature observed by commercial aircraft, J. Atmos. Sci., 42, 950-960, 1985.

Neale, R. B. and Hoskins, B. J.: A standard test for AGCMs including their physical parametrizations: I: The proposal, Atmos. Sci. Lett., 1, 101-107, doi:10.1006/asle.2000.0022, 2000.

Neale, R. B., Chen, C.-C., Gettelman, A., Lauritzen, P. H., Park, S., Williamson, D. L., Conley, A. J., Garcia, R., Kinnison, D., Lamarque, J.-F., Marsh, D., Mills, M., Smith, A. K., Tilmes, S., Vitt, F., Morrison, H., Cameron-Smith, P., Collins, W. D., Iacono, M. J., Easter, R. C., Ghan, S. J., Liu, X., Rasch, P. J., and Taylor, M. A.: Description of the NCAR Community Atmosphere Model (CAM 5.0), NCAR Technical Note NCAR/TN-
486+STR, National Center for Atmospheric Research, Boulder, Colorado, 268, 2010.

O'Gorman, P. A. and Schneider, T.: The hydrological cycle over a wide range of climates simulated with an idealized GCM, J. Climate, 21, 3815-3832, 2008.

Park, S. and Bretherton, C. S.: The University of Washington shallow convection and moist turbulence schemes and their impact on climate simulations with the Community Atmosphere Model, J. Climate, 22, 3449-3469, doi:10.1175/2008JCLI2557.1, 2009.

Petrik, R., Baldauf, M., Schlünzen, H., and Gassmann, A.: Tropospheric response to stratospheric perturbations in a relatively simple general circulation model, Tellus A, 63, 707-726, doi:10.1111/j.1600-0870.2011.00528.x, 2011.

Polvani, L. M. and Kushner, P. J.: Tropospheric response to stratospheric perturbations in a relatively simple general circulation model, Geophys. Res. Lett., 29, 1114 doi:10.1029/2001GL014284, 2002.

Pond, S., Fissel, D. B., and Paulson, C. A.: A note on bulk aerodynamic coefficients for sensible heat and moisture fluxes, Bound.Lay. Meteorol., 6, 333-339, 1974.

Rasch, P. J., Coleman, D. B., Mahowald, N., Williamson, D. L., Lin, S.-J., Boville, B. A., and Hess, P.: Characteristics of atmospheric transport using three numerical formulations for atmospheric dynamics in a single GCM framework, J. Climate, 19, 2243-2266, doi:10.1175/JCLI3763.1, 2006.

Rauscher, S. A., Ringler, T. D., Skamarock, W. C., and Mirin, A. A.: Exploring a global multi-resolution modeling approach using aquaplanet simulations, J. Climate, 26, 2432-2452, doi:10.1175/JCLI-D-12-00154.1, 2013.

Reed, K. A. and Jablonowski, C.: Idealized tropical cyclone simulations of intermediate complexity: a test case for AGCMs, J. Adv. Model. Earth Syst., 4, M04001, doi:10.1029/2011MS000099, 2012.

Reed, K. A., Bacmeister, J. T., Rosenbloom, N. A., Wehner, M. F., Bates, S. C., Lauritzen, P. H., Truesdale, J. E., and Hannay, C.: Impact of the dynamical core on the direct simulation of tropical cyclones in a high-resolution global model, Geophys. Res. Lett., 42, 3603-3608, doi:10.1002/2015GL063974, 2015.

Richardson, M. I., Toigo, A. D., and Newman, C. E.: PlanetWRF: a general purpose, local to global numerical model for planetary atmospheric and climate dynamics, J. Geophys. Res.-Planet., 112, E09001, doi:10.1029/2006JE002825, 2007.

Saha, S., Moorthi, S., Pan, H.-L., Wu, X., Wang, J., Nadiga, S., Tripp, P., Kistler, R., Woollen, J., Behringer, D., Liu, H., Stokes, D., Grumbine, R., Gayno, G., Wang, J., Hou, Y.-T., Chuang, H.-Y., Juang, H.-M. H., Sela, J., Iredell, M., Treadon, R., Kleist, D., van Delst, P., Keyser, D., Derber, J., Ek, M., Meng, J., Wei, H., Yang, R., Lord, S., van den Dool, H., Kumar, A., Wang, W., Long, C., Chelliah, M., Xue, Y., Huang, B., Schemm, J.-K., Ebisuzaki, W., Lin, R., Xie, P., Chen, M., Zhou, S., Higgins, W., Zou, C.-Z., Liu, Q., Chen, Y., Han, Y., Cucurull, L., Reynolds, R. W., Rutledge, G., and Goldberg, M.: The NCEP climate forecast system reanalysis, B. Am. Meteorol. Soc., 91, 1015-1057, 2010.

Schneider, T., Bischoff, T., and Płotka, H.: Physics of changes in synoptic midlatitude temperature variability, J. Climate, 28 , 2312-2331, 2015. 
Semane, N. and Bechtold, P.: Convection and waves on small earth and deep atmosphere, Tellus A, 67, 25151, doi:10.3402/tellusa.v67.25151, 2015.

Simmons, A. J. and Burridge, D. M.: An energy and angularmomentum conserving vertical finite-difference scheme and hybrid vertical coordinates, Mon. Weather Rev., 109, 758-766, doi:10.1175/1520-0493(1981)109<0758:AEAAMC>2.0.CO;2, 1981.

Skamarock, W. C.: Evaluating mesoscale NWP models using kinetic energy spectra, Mon. Weather Rev., 132, 3019-3032, 2004.

Skamarock, W. C.: Kinetic energy spectra and model filters, in: Numerical Techniques for Global Atmospheric Models, Springer, 495-512, doi:10.1007/978-3-642-11640-7_14, 2011.

Skamarock, W. C., Klemp, J. B., Dudhia, J., Gill, D. O., Barker, D. M., Duda, M. G., Huang, X.-Y., Wang, W., and Powers, J. G.: A description of the Advanced Research WRF Version 3, NCAR Tech. Note NCAR/TN-475+STR, National Center for Atmospheric Research, Boulder, Colorado, 113 pp., 2008.

Smedman, A.-S., Sahlée, U., Högström, E., and Johansson, C.: Critical re-evaluation of the bulk transfer coefficient for sensible heat over the ocean during unstable and neutral conditions, Q. J. Roy. Meteor. Soc., 133, 227-250, 2007.

Smolarkiewicz, P. K., Margolin, L. G., and Wyszogrodzki, A. A.: A class of nonhydrostatic global models, J. Atmos. Sci., 58, 349-364, doi:10.1175/15200469(2001)058<0349:ACONGM>2.0.CO;2, 2001.

Taylor, M. A. and Fournier, A.: A compatible and conservative spectral element method on unstructured grids, J. Comput. Phys., 229, 5879-5895, doi:10.1016/j.jcp.2010.04.008, 2010.

Taylor, M., Tribbia, J., and Iskandarani, M.: The spectral element method for the shallow water equations on the sphere, J. Comput. Phys., 130, 92-108, doi:10.1006/jcph.1996.5554, 1997.

Thurre, C.: Etude de l'ajustement hydrostatique suite à un forçage diabatique dans un modèle pleinement élastique, $\mathrm{PhD}$ dissertation, McGill University, Department of Atmospheric and Oceanic Sciences, Montreal, Canada, 124 pp., 1998.

Thurre, C. and Laprise, R.: Projecting diabatic heating onto hydrostatic modes in a non-hydrostatic model. Research Activities in Atmospheric and Oceanic Modelling, WMO, WMO/TD-No. 734, Report No. 23, 2.66-2.68, 1996.

Tomita, H. and Satoh, M.: A new dynamical framework of nonhydrostatic global model using the icosahedral grid, Fluid Dyn. Res., 34, 357-400, doi:10.1016/j.fluiddyn.2004.03.003, 2004.

Ullrich, P. A., Melvin, T., Jablonowski, C., and Staniforth, A.: A proposed baroclinic wave test case for deep- and shallowatmosphere dynamical cores, Q. J. Roy. Meteor. Soc., 140, 15901602, doi:10.1002/qj.2241, 2014.

Wan, H., Giorgetta, M. A., and Bonaventura, L.: Ensemble HeldSuarez test with a spectral transform model: variability, sensitivity, and convergence, Mon. Weather Rev., 136, 1075-1092, doi:10.1175/2007MWR2044.1, 2008.

Wan, H., Rasch, P. J., Taylor, M. A., and Jablonowski, C.: Shortterm time step convergence in a climate model, J. Adv. Model. Earth Syst., 7, 215-225, doi:10.1002/2014MS000368, 2015.

Weber, S. L.: The utility of Earth system models of intermediate complexity (EMICs), Wiley Interdisciplinary Reviews: Climate Change, 1, 243-252, 2010.
Wedi, N. P. and Smolarkiewicz, P. K.: A framework for testing global non-hydrostatic models, Q. J. Roy. Meteor. Soc., 135, 469-484, doi:10.1002/qj.377, 2009.

Wheeler, M. and Kiladis, G. N.: Convectively coupled equatorial waves: analysis of clouds and temperature in the wavenumber-frequency domain, J. Atmos. Sci., 56, 374-399, doi:10.1175/1520-0469(1999)056<0374:CCEWAO>2.0.CO;2, 1999.

Whitehead, J. P., Jablonowski, C., Rood, R. B., and Lauritzen, P. H.: A stability analysis of divergence damping on a latitude-longitude grid, Mon. Weather Rev., 139, 2976-2993, doi:10.1175/2011MWR3607.1, 2011.

Williamson, D. L.: Time-split versus process-split coupling of parameterizations and dynamical core, Mon. Weather Rev., 130, 2779-2799, 2002.

Williamson, D. L.: Convergence of aqua-planet simulations with increasing resolution in the Community Atmospheric Model, Version 3, Tellus A, 60, 848-862, doi:10.1111/j.16000870.2008.00339.x, 2008a.

Williamson, D. L.: Equivalent finite volume and Eulerian spectral transform horizontal resolutions established from aquaplanet simulations, Tellus A, 60, 839-847, doi:10.1111/j.16000870.2008.00340.x, 2008b.

Williamson, D. L.: The effect of time steps and time-scales on parametrization suites, Q. J. Roy. Meteor. Soc., 139, 548-560, 2013.

Williamson, D. L. and Olson, J. G.: Dependence of aqua-planet simulations on time step, Q. J. Roy. Meteor. Soc., 129, 2049-2064, doi:10.1256/qj.02.62, 2003.

Williamson, D. L., Blackburn, M., Hoskins, B. J., Nakajima, K., Ohfuchi, W., Takahashi, Y. O., Hayashi, Y. Y., Ishiwatari, H. N. M., McGregor, J., Borth, H., Wirth, V., Frank, H., Bechtold, P., Wedi, N. P., Tomita, H., Satoh, M., Zhao, M., Held, I. M., Suarez, M. J., Lee, M.-I., Watanabe, M., Kimoto, M., Liu, Y., Wang, Z., Molod, A., Rajendran, K., Kitoh, A., and Stratton, R.: The APE Atlas National Center for Atmospheric Research, NCAR Technical Note, NCAR/TN-484+ STR, 508 pp., doi:10.5065/D6FF3QBR, 2012.

WMO: International Meteorological Vocabulary, 2nd Edn., World Meteorological Organization, Geneva, Switzerland, Vol. 182, p. 784, 1992.

Yao, W. and Jablonowski, C.: Spontaneous QBO-like oscillations in an atmospheric model dynamical core, Geophys. Res. Lett., 40, 3772-3776, doi:10.1002/grl.50723, 2013.

Yao, W. and Jablonowski, C.: Idealized quasi-biennial oscillations in an ensemble of dry GCM dynamical cores, J. Atmos. Sci., 72, 2201-2226, doi:10.1175/JAS-D-14-0236.1, 2015.

Zhang, G. J. and McFarlane, N. A.: Role of convective scale momentum transport in climate simulation, J. Geophys. Res., 100, 1417-1426, doi:10.1029/94JD02519, 1995.

Zhang, H., Zhang, M., and Zeng, Q.-C.: Sensitivity of simulated climate to two atmospheric models: Interpretation of differences between dry models and moist models, Mon. Weather Rev., 141, 1558-1576, 2013. 